\title{
Nonlinear ultrafast acoustics at the nano scale
}

\author{
P.J.S. van Capel ${ }^{\mathrm{a}, *}$, E. Péronne ${ }^{\text {b,c }}$, J.I. Dijkhuis ${ }^{\text {a }}$
}

a Debye Institute for Nanomaterials Science, Center for Extreme Matter and Emergent
Phenomena, Utrecht University, P.O. Box 80000, 3508 TA Utrecht, The Netherlands
b CNRS, UMR 7588, Institut des NanoSciences de Paris, F-75005 Paris, France
c Sorbonne Universités, UPMC Univ. Paris 06, UMR 7588, INSP, F-75005 Paris, France

Pulsed femtosecond lasers can generate acoustic pulses propagating in solids while displaying either dif-fraction, attenuation, nonlinearity and/or dispersion. When acoustic attenuation and diffraction are neg-ligible, shock waves or solitons can form during propagation. Both wave types are phonon wavepackets with characteristic length scales as short as a few nanometer. Hence, they are well suited for acoustic characterization and manipulation of materials on both ultrafast and ultrashort scales. This work pre-sents an overview of nonlinear ultrasonics since its first experimental demonstration at the beginning of this century to the more recent developments. We start by reviewing the main properties of nonlinear ultrafast acoustic propagation based on the underlying equations. Then we show various results obtained by different groups around the world with an emphasis on recent work. Current issues and directions of future research are discussed. 


\section{Introduction}

Whenever light cannot enter matter, sound becomes the first choice for imaging buried structures. Megahertz ultrasound techniques have been extensively studied in the past and gave birth to numerous applications such as sonography and nondestructive testing with a resolution of the order of a few micron.

The development of picosecond and femtosecond pulsed lasers in the mid-80's [1] opened the way to all-optical pump probe techniques to study acoustic waves. The archetypal experimental arrangement Fig. 1(a) is nowadays referred to as picosecond ultrasonics [2] or ultrafast acoustics. A short optical 'pump' pulse heats a (generally metallic) transducer material. Through the thermoelastic effect (the heated material exerts a sudden stress to its surroundings), a coherent longitudinal strain pulse $\eta(z, t)$ is formed. The acoustic wave reflects at the transducer boundaries, locally modifying the optical properties. Such modifications are measured by a delayed optical 'probe' pulse. For a review of developments in detection techniques, we refer to another contribution in this issue.

This technique allows the generation of acoustic pulses as short as a few picosecond, with a bandwidth typically $100 \mathrm{GHz}$, at strain amplitudes of the order of $10^{-5}-10^{-4}$. The principle was first demonstrated and theoretically analyzed for metal films and semiconductors $[3,4,1]$. Since the sound velocity in semiconductors and metals is typically several $\mathrm{nm} / \mathrm{ps}$, picosecond acoustic pulses extend spatially up to several tens of nanometers.

In standard ultrafast acoustics relying on the thermoelastic effect, the range of accessible phonon frequencies is determined by the properties of the generator material, and is limited by either the optical penetration depth or the electron diffusion length (the typical depth over which heat is deposited) $[5,6]$.

To really extend application of ultrasound to the study and manipulation of nanostructures, acoustic frequencies in the $100 \mathrm{GHz}-1 \mathrm{THz}$ range are required; an order of magnitude higher than generated by conventional techniques. However, very few techniques can generate such acoustic frequencies. They are mainly based on transient optical excitation by laser pulses.

In some cases, $\mathrm{THz}$ frequencies have been generated by optically exciting vibrational modes of a very thin single layer or carefully designed multilayers [7-12]. These heterostructures allow some control over the properties of the generated coherent wave [13]. In strained quantum well structures where strain is generated through piezeoelectric screening, tunable phonon generation with a bandwidth of several hundreds of $\mathrm{GHz}$ has been demonstrated $[14,15]$, with absolute strain amplitudes up to $2 \%$ [16]. A recent paper has shown that by generation in the semiconductor itself broad, and high-amplitude, tunable strain waves can be generated [17]. More information may be found in other contributions in this special issue.

High acoustic frequencies can also be reached by using the intrinsic acoustic nonlinearity of crystals. This does not require the design of specific transducers and (in sharp contrast with nanostructured transducers) results in a broadband acoustic spectrum. We report in this paper on nonlinear ultrasonics, the nonlinear propagation of acoustic pulses in lattices, in the framework of femtosecond laser ultrasonics.

Section 2 is an introduction to the propagation equation in anisotropic solids which includes dispersion, diffraction, viscous damping and nonlinearity. The equation is rather complex and impractical to solve mainly because of its tensorial nature. However, making some simplifying assumptions relevant to most nonlinear ultrasonic experiments, the propagation equation is rewritten into an analytical form which we will label the cylindrical Korteweg-de Vries-Burgers equation (cKdVB).
In the remainder of the paper, we will discuss specific regimes, in which one or more of the terms in the cKdVB equation can be neglected. Section 3 treats the linear regime, where nonlinear effects are negligible, to illustrate the effects of the three linear terms (dispersion, diffraction, damping) in the governing equation.

Nonlinearity modifies the acoustic pulse shape and leads to frequency upconversion. Section 5 is devoted to the case when dispersion and diffraction are negligible, and acoustic shock waves are formed, see Fig. 1(b) $[18,19]$.

The group of H.J. Maris was the first to demonstrate the nonlinear propagation of picosecond ultrasonic pulses [20] in $\mathrm{SiO}_{2}, \mathrm{Si}, \mathrm{Al}_{2} \mathrm{O}_{3}$, and $\mathrm{MgO}$ in 2001, leading to acoustic wavelengths shorter than the typical wavelength at generation. When nonlinearity is counterbalanced by acoustic dispersion, acoustic solitons can form, see Fig. 1(c). These solitons have intriguing properties. Once formed, they show a remarkable resilience to deformation and attenuation, for example by energy transfer to an electronic two-level system [21] or by anharmonic phonon decay [22], and propagate unperturbed over distances as large as several $\mathrm{mm}$ [23]. The group of J.I. Dijkhuis has demonstrated the existence of a $0.87 \mathrm{THz}$ component in the spectrum of the acoustic pulse after nonlinear propagation in ruby by means of two-level phonon spectroscopy [21,24].

Acoustic solitons have also been studied by time-resolved reflectometric [25,26] and interferometric [27,28] methods. Although this paper focuses on bulk propagation, it is good to mention that nonlinear propagation of surface waves has been reported as well by beam deflection techniques $[29,30]$. We will treat nonlinear dispersive propagation in Section 6.

Section 7 addresses current experimental challenges and sketches future directions of research.

Throughout this paper, we will illustrate phenomena by numerical computations and experimental results. The numerical approach we have developed is presented in Section 2. We will first present the setups with which experimental results have been obtained.

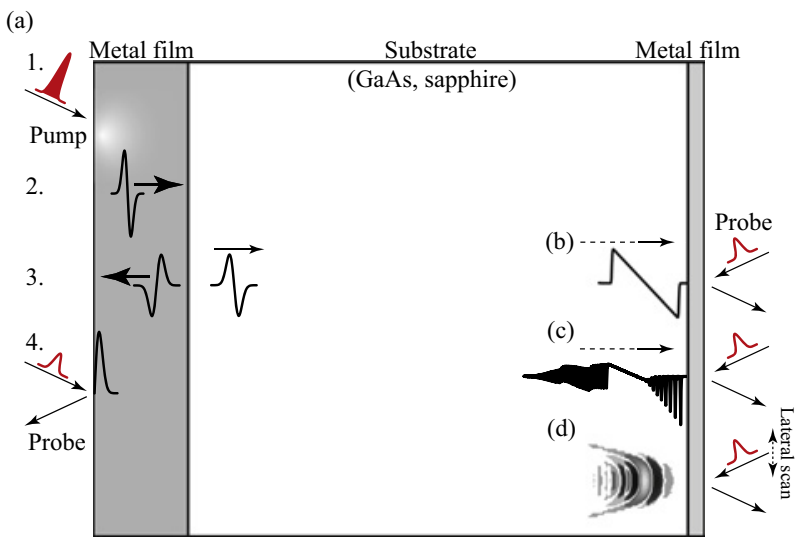

Fig. 1. (a) Picosecond ultrasonics technique. 1. Absorption of energy from pump beam, and heating of the surface region. Coherent strain is generated by thermal expansion of the lattice. 2. The generated strain travels into the film. 3. Part of the wave is reflected at the metal/substrate interface. 4 . The part that returns to the interface is detected by a delayed, weak probe pulse. (b) Propagation of the highamplitude acoustic wave launched into the substrate at room temperature: due to nonlinearity, the wave transforms into a $\mathrm{N}$ - or shock wave. The shock wave is detected at the other side by the same technique as in (a). (c) Like (b) but now at low temperatures: the wave develops into a train of solitons, and high-frequency tail. (d) Sketch of diffraction of a soliton train monitored by lateral scanning of the probe beam; brightness indicates strain amplitude. 


\subsection{Experimental arrangements}

The various aspects of ultrafast acoustic pulse propagation are illustrated by experimental results obtained on sapphire samples (in Utrecht) and gallium arsenide samples (in Paris). These samples are typically several hundreds of $\mu \mathrm{m}$ thick, so that nonlinearity can take effect. The Paris samples are covered with typically $40 \mathrm{~nm}$ thick Al films on both sides, where one film acts as generator and one as detector. This way, the wave profile after traversal of the sample can be monitored. The Utrecht samples are covered with $\mathrm{Cr}$ films as generator/detector.

The current standard detection technique in picosecond ultrasonics is interferometry, either in single-shot [31] or non-destructive mode $[32,33,5,34]$, supplying information on both the amplitude and phase of the reflected optical pulse. The reflected light amplitude is modulated by the acoustic pulse in the detector film [1], and the resolution is therefore limited to the optical penetration depth. The phase, however, is modulated by the shift of the detector film surface due to reflection of the acoustic pulse, and in principle yields higher temporal resolution. The time resolution of both setups is then limited by the optical pulse durations and of the order of $200 \mathrm{fs}$.

The Paris setup is based on a conventional high-repetition rate femtosecond laser system coupled to lock-in phase detection, and operates at temperatures from room temperature down to $15 \mathrm{~K}$. The pump beam is modulated up to $1.8 \mathrm{MHz}$ for improved sensitivity of the relative change of reflectivity which is measured with a Sagnac [27] or Michelson [35] interferometer. The set-up allows measurements of amplitude as well as phase change of down to $10^{-7}$ (i.e. surface displacement as small as $10 \mathrm{fm}$ ). Moreover, the surface of the sample can be laterally scanned. However, the energy per pump pulse is limited up to around $13 \mathrm{~nJ}$ which implies initial strains as high as $10^{-3}$ for pump beam diameters of the order of $10 \mu \mathrm{m}$.

The Utrecht setup uses a $1 \mathrm{kHz}$ amplified femtosecond laser system in connection with a Sagnac interferometer [34], and can be operated from room temperature down to $10 \mathrm{~K}$. Strains several times $10^{-3}$ can be generated by pumping the generator film close to its damage threshold. At the same time, the pump beam sizes can be an order of magnitude larger $(200 \mu \mathrm{m})$ compared to the $\sim 10 \mu \mathrm{m}$ of conventional femtosecond systems required to reach nonlinear conditions. The sensitivity is typically good enough to observe relative effects on the order of $10^{-5}$, corresponding to surface displacements of $1 \mathrm{pm}$.

\section{Acoustic propagation in lattice}

Lattices are a periodic ordering of atoms which from an acoustic point of view can be modeled by masses linked by springs. Given the atomic displacement $u_{i}(i=x, y, z)$, the principle of dynamics assuming a harmonic atomic potential leads to the generalized Hooke's law:

$\rho \partial_{t}^{2} u_{i}=C_{i j k l} \partial_{j k}^{2} u_{l}$

where $C_{i j k l}$ is the stiffness tensor and $\rho$ is the crystalline density of the unstrained solid. Eq. (1) predicts linear, undamped harmonic wave propagation.

In nonlinear ultrasonics, this approximation is not valid anymore. First, the amplitude of the displacement can be large enough such that the interaction between the lattice sites is becoming anharmonic and higher order elastic effects come into play [36]. Second, because of the periodicity of the lattice, it is well known that the sound velocity (i.e. elastic constant) becomes frequency dependent at acoustic wavelengths approaching the lattice constant [37]. Both effects have been studied in the famous
Fermi-Pasta-Ulam numerical experiment which can be regarded as the first realization of an acoustic soliton in crystal lattices [38]. Third, viscous damping cannot be neglected at high frequencies.

The aim of this section is to expand on the theoretical background of the nonlinear ultrafast acoustic experiments which have led to high acoustic frequency generation and shock wave and soliton formation. Since most crystals are anisotropic media, all relevant parameters are usually tensors and the propagation equation is tensorial. Fortunately, most of the ultrasonics experiments deal with ultrashort longitudinal acoustic pulses which can be described using a simplified, quasi-1D version of the propagation equation. This description explicitly takes into account diffraction, a fourth effect influencing the acoustic pulse shape.

\subsection{Nonlinear propagation equation}

Following the work of Hao and Maris [20], the propagation equation Eq. (1) is re-evaluated when the internal energy dependence with strain follows a power series [39]. At the lowest nonlinear order, $C_{i j k l}$ in Eq. (1) must be replaced by $C_{i j k l}+\widetilde{C}_{i j k l m p} \partial_{m} u_{p}$ with

$\widetilde{C}_{i j k l m p}=C_{k j m p} \delta_{i l}+C_{m j k l} \delta_{i p}+C_{i j l m} \delta_{l p}+C_{i j k l m p}$.

According to this equation the nonlinearity has two different origins: one arises from geometry (the three second order terms) and the other from the anharmonicity of the interaction between the atoms in the lattice (the third order term $C_{i j k l m p}$ ) following Brugger's definition [40]. In the general case the elastic coefficients are frequency dependent since crystalline solids are dispersive. When the acoustic wavelength significantly exceeds the lattice parameter, one way to take into account the dispersion is to allow for a small momentum dependence for the elastic coefficient such as $C_{i j l m}(k)=C_{i j l m}(k=0)+i D_{i j l m \zeta} k_{\varsigma}-F_{i j l m \zeta v} k_{\varsigma} k_{v}$ [41]. The coefficient $D$ is nonzero mainly for transverse waves propagating along a low-symmetry axis. The parameter $F$ is usually positive, an explicit expression of normal dispersion.

Attenuation is a serious issue in ultrafast laser ultrasonic experiments since the acoustic pulse contains phonon frequencies which may be scattered strongly during propagation over several microns. Phonon scattering in crystals is a rather complex phenomenon which can involve impurities, phonon-phonon scattering as well as surface/interface scattering, leading to a convoluted law of attenuation depending on lattice temperature and phonon frequency. The simplest approximation is to assume viscous damping (Stokes law), such that the sound attenuation is proportional to the square of the frequency. It is equivalent to introducing a dynamic viscosity $\mu_{i j k l}$. After these modifications, the nonlinear equation for ultrashort acoustic pulses in crystals reads

$\rho \partial_{t}^{2} u_{i}=\left[C_{i j k l}+\widetilde{C}_{i j k l m p} \partial_{m} u_{p}+D_{i j k l \varsigma} \partial_{\varsigma}+F_{i j k l \zeta v} \partial_{\varsigma} \partial_{\nu}+\mu_{i j k l} \partial_{t}\right] \partial_{j k}^{2} u_{l}$.

In practice, Eq. (3) is never solved fully in order to understand experimental results since most ultrafast acoustic experiments deal with longitudinal acoustic pulses in a single propagation direction. However Eq. (3) reminds us of the importance of the direction of propagation and the polarization of the pulse in order to understand the nonlinear behavior of ultrashort acoustic pulses.

\subsection{Korteweg-de Vries-Burgers equation in paraxial approximation}

As was already stated in the introduction, ultrashort acoustic pulses are mostly generated by thin metallic transducers or heterostructures. When the main transduction mechanism is thermal stress induced by optical absorption, the longitudinal component of the stress is much larger than the transverse one. More importantly, the nonlinear effect is always cumulative for longitudinal 
waves: these interact nonlinearly which other longitudinal waves producing again longitudinal waves. This is not generally the case for transverse waves in lattices.

Since the lateral extension of the acoustic pulse in the $x-y$ directions (order $\mu \mathrm{m}$ ) is given by the laser spot and much larger than the in-depth extension (order $\mathrm{nm}$ ), the acoustic wavevector is mainly parallel to the propagation direction $z\left(k_{z} \gg k_{x, y}\right)$.

We can therefore reduce the problem to quasi-1D propagation of longitudinal strain along the $z$ direction. First, since $k_{z} \gg k_{x, y}$ the nonlinear and dispersive terms in Eq. (3) are assumed to work only along the $z$ direction, i.e. $i=z$. Second, the linear term in Eq. (3) is treated within the paraxial approximation [42]. For convenience, we switch from a Cartesian $x, y, z$ to cylindrical $r, \theta, z$ coordinate system.

Because nonlinear ultrafast acoustics deals with propagating strain pulses, it is convenient to focus on the one direction version of Eq. (3). We first introduce the strain $\eta(z, r, \theta, t)=\partial_{z} u$. Second, this strain is evaluated in a comoving frame $\left(t \rightarrow t\right.$ and $z \rightarrow z-v_{s} t$ with $v_{s}=\sqrt{C_{z z} / \rho}$ the longitudinal sound velocity). Third, by applying the slow envelope approximation $\left(\partial_{t}^{2} \eta, \partial_{z} \partial_{t} \eta \sim 0\right)$ and integrating once over $z$, we finally find

$$
\begin{aligned}
\partial_{t} \eta= & -\frac{C_{N L}}{2 v_{s} \rho} \eta \partial_{z} \eta-\frac{F}{2 v_{s} \rho} \partial_{z z z}^{3} \eta+\frac{\mu}{2 \rho} \partial_{z z}^{2} \eta-\frac{\xi^{2}(\theta) v_{s}}{2} \frac{1}{r} \partial_{r} r \partial_{r} \\
& \times \int_{-\infty}^{z} \eta d z^{\prime} .
\end{aligned}
$$

Here, $F$ is the dispersion coefficient and $C_{N L}$ is the effective nonlinear elastic coefficient (negative in most crystals). To the lowest order, the paraxial approximation leads to the renormalization of the transverse Laplacian by a diffraction strength factor $\xi^{2}(\theta)$ depending on the propagation direction, the polarization and the lattice symmetry $[42,26]$. This factor is angle dependent due to the elastic anisotropy in arbitrary lattices [43]. For small angles with respect to the $z$ axis, the dependence on $\theta$ will be negligible and cylindrical symmetry can be assumed. All coefficients in Eq. (4) depend on lattice properties and propagation direction.

When looking for numerical solutions of Eq. (4) it is convenient to rewrite it such that all variables take on values close to unity. Let us redefine the coefficients the following way:

- $t \rightarrow T_{\text {prop }} t$ where $T_{\text {prop }}=\frac{d}{v_{s}}$ is the propagation time through the sample over a distance $d$,

- $r \rightarrow r_{0} r$ where $r_{0}$ is the laser spot size on the transducer,

- $\eta \rightarrow \eta_{0} \eta$ where $\eta_{0}$ is the amplitude of the initial strain $\eta(z, t=0)$,

- $z \rightarrow z_{0} z$ where $z_{0}$ is the length of the initial strain pulse $\eta(z, t=0)$,

such that Eq. (4) takes the new form

$\partial_{t} \eta=\frac{T_{\text {prop }}}{T_{n l}} \eta \partial_{z} \eta-\frac{T_{\text {prop }}}{T_{\text {disp }}} \partial_{z z z}^{3} \eta+\frac{T_{\text {prop }}}{T_{\text {att }}} \partial_{z z}^{2} \eta-\frac{T_{\text {prop }}}{T_{\text {diff }}} \frac{1}{r} \partial_{r} r \partial_{r} \int_{-\infty}^{z} \eta d z^{\prime}$,

with $T_{n l}=-\frac{2 v_{s} \rho z_{0}}{C_{N L} \eta_{0}}, T_{\text {disp }}=\frac{2 \rho v_{s} z_{0}^{3}}{F}, T_{\text {att }}=\frac{2 \rho z_{0}^{2}}{\mu}$ and $T_{\text {diff }}=\frac{2 r_{0}^{2}}{\xi^{2} z_{0} v_{s}}$ the typical times it takes for respectively nonlinearity, dispersion, attenuation and diffraction to significantly alter the shape of the initial pulse.

It appears clearly that the different effects scale with the lattice properties and the strain profile before propagation. The influence of the initial strain profile on the propagation scenario may be emphasized by judicious definition of the parameters $\eta_{0}, z_{0}$ and $r_{0}$.

A natural choice of $\eta_{0}$ is the strain amplitude such that $|\eta(z, r, 0)|$ has a maximum value of 1 . It is then natural to define $z_{0}$ such that the nonlinear term is normalized as well. Imposing $\left|\eta(z, r, 0) \partial_{z} \eta(z, r, 0)\right| \leqslant 1$ leads to the definition $z_{0}=\frac{\eta_{0}}{\left|\partial_{z} \eta\right|_{z=z_{s}}}$ with $z_{s}$ the position of the stiffest part of the initial pulse. After this renor- malization, the amplitude of the nonlinear term at $t=0$ is then entirely determined by the constant $T_{n l}$.

In nonlinear (and nondissipative) acoustics, it is common to define the shock distance $L_{s h o c k}=\frac{2 \rho v_{s}^{2}}{\left|C_{N L}\right|\left|\partial_{z} \eta\right|_{z=z_{s}}}$ by the shortest propagation length for which part of the wave front acquires an infinite slope (i.e. a shock front). With the help of $z_{0}$ as defined previously one can verify that $L_{\text {shock }}=v_{s} T_{n l}$ which means that $T_{n l}$ can be interpreted as the time it take for the pulse to shock. This is confirmed by the computation of the strain pulse at $t=T_{n l}$ as displayed in Figs. 2 and 3.

Following the same idea, the radius normalization can be defined as $r_{0}^{2}=\frac{\eta_{0}}{\max \left|\frac{1}{r} \partial_{r} r_{r} \eta\right|}$. For a Gaussian laser profile, $r_{0}$ is half the radius at $\frac{1}{e}$. There is no parameter left to normalize dispersion and attenuation in the same way. Furthermore, because of the higher order derivatives $T_{\text {att }}$ and $T_{\text {disp }}$ are associated to other time scales which also depend on the initial profile. We will in particular see in Section 4 that nonlinearity increases the acoustic frequencies in the strain pulse, which in turn will enhance dispersion and attenuation. It is then necessary to use a numerical approach to evaluate the terms against each other.

Eq. (4) is a $(3+1)$-dimensional Korteweg-de Vries-Burgers (KdVB) equation in cylindrical coordinates, taking into account diffraction in the paraxial approximation. We therefore denominate Eq. (4) the cylindrical Korteweg-de Vries-Burgers equation (cKdVB). Note that Eq. (4) reduces to well known equations, depending on the approximation:

- The Kadomtsev-Petviashvili (KP) equation when $\frac{T_{\text {prop }}}{T_{\text {att }}} \rightarrow 0$ [44].

- The Korteweg-de Vries (KdV) equation when $\frac{T_{\text {prop }}}{T_{\text {att }}} \rightarrow 0$ and $\frac{T_{\text {prop }}}{T_{\text {diff }}} \rightarrow 0$.

- The Khokhlov-Zabolotskaya-Kouznetsov (KZK) equation when $\frac{T_{\text {prop }}}{T_{\text {disp }}} \rightarrow 0$ [45].

- The Burgers equation when $\frac{T_{\text {prop }}}{T_{\text {disp }}} \rightarrow 0$ and $\frac{T_{\text {prop }}}{T_{\text {diff }}} \rightarrow 0$.

Thanks to the abundant literature on those equations, many features of the more general cKdVB equation may be inferred. For example, the KdV equation is well known for its special traveling wave solutions called solitons. The KP equation also admits soliton-like solutions call line solitons, as nicely demonstrated by tidal waves in shallow sea shores [46].

\subsection{Numerical solution of cylindrical KdVB}

2D and 3D nonlinear propagation of ultrashort strain pulses has been modelized and computed in previous works. Kawasaki et al. [47] compared numerically different models for ultrafast acoustics to study the effect of the initial conditions on the solitons. Muskens and Dijkhuis computed the soliton shape and the dispersive tail behavior under diffraction conditions [24]. In addition to these works, we explicit a simple numerical scheme to solve Eq. (5) before comparing computed strain pulses with measured ones.

When diffraction can be neglected, a composite Runge-Kutta algorithm (CRK43) is widely used by the nonlinear ultrafast acoustics community [24]. It is based on a spectral decomposition where the low-frequency strain components are treated with the help of an implicit scheme and the high frequency components with an explicit scheme [48]. This allows to solve the KdV and Burgers equations in the Fourier domain. ${ }^{1}$

The diffraction term can itself be solved numerically by different kinds of algorithms like a finite difference (FD) scheme (either explicit or implicit) [24]. Since Eq. (5) including the diffraction term is only exactly solvable with the help of a Hankel

\footnotetext{
${ }^{1}$ The Fourier transform is defined as $g(q)=\frac{1}{2 \pi} \int_{-\infty}^{+\infty} f(z) e^{-i q z} d z$.
} 

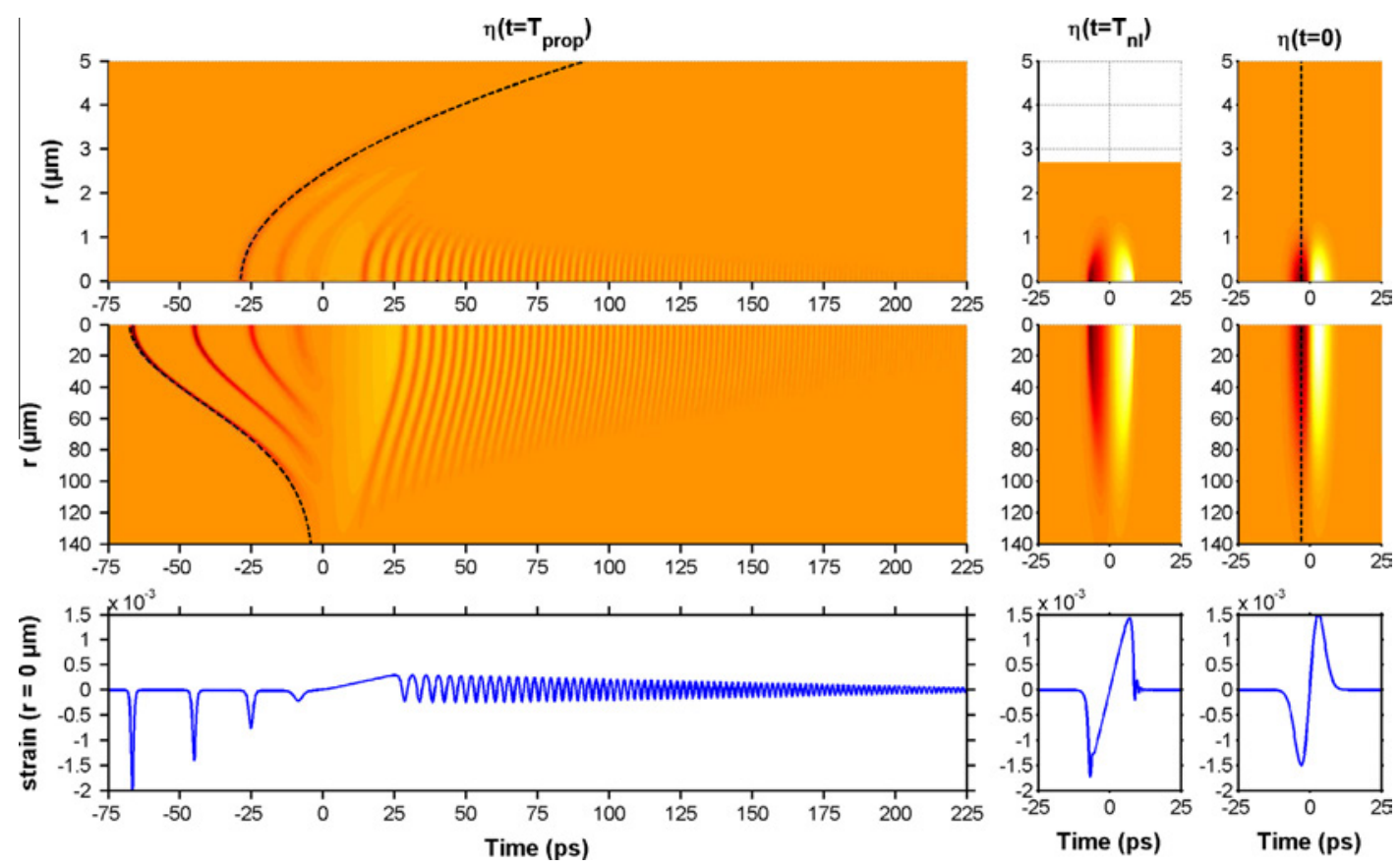

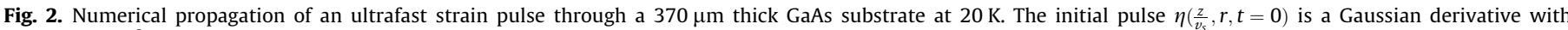

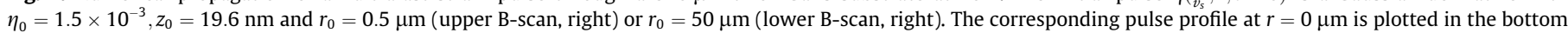

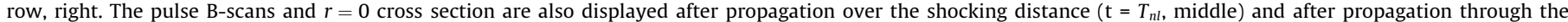

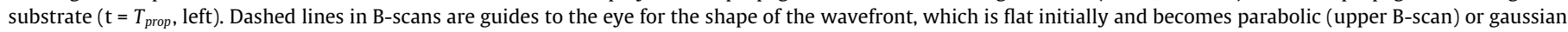
(lower B-scan) depending on diffraction to be important or not, respectively.
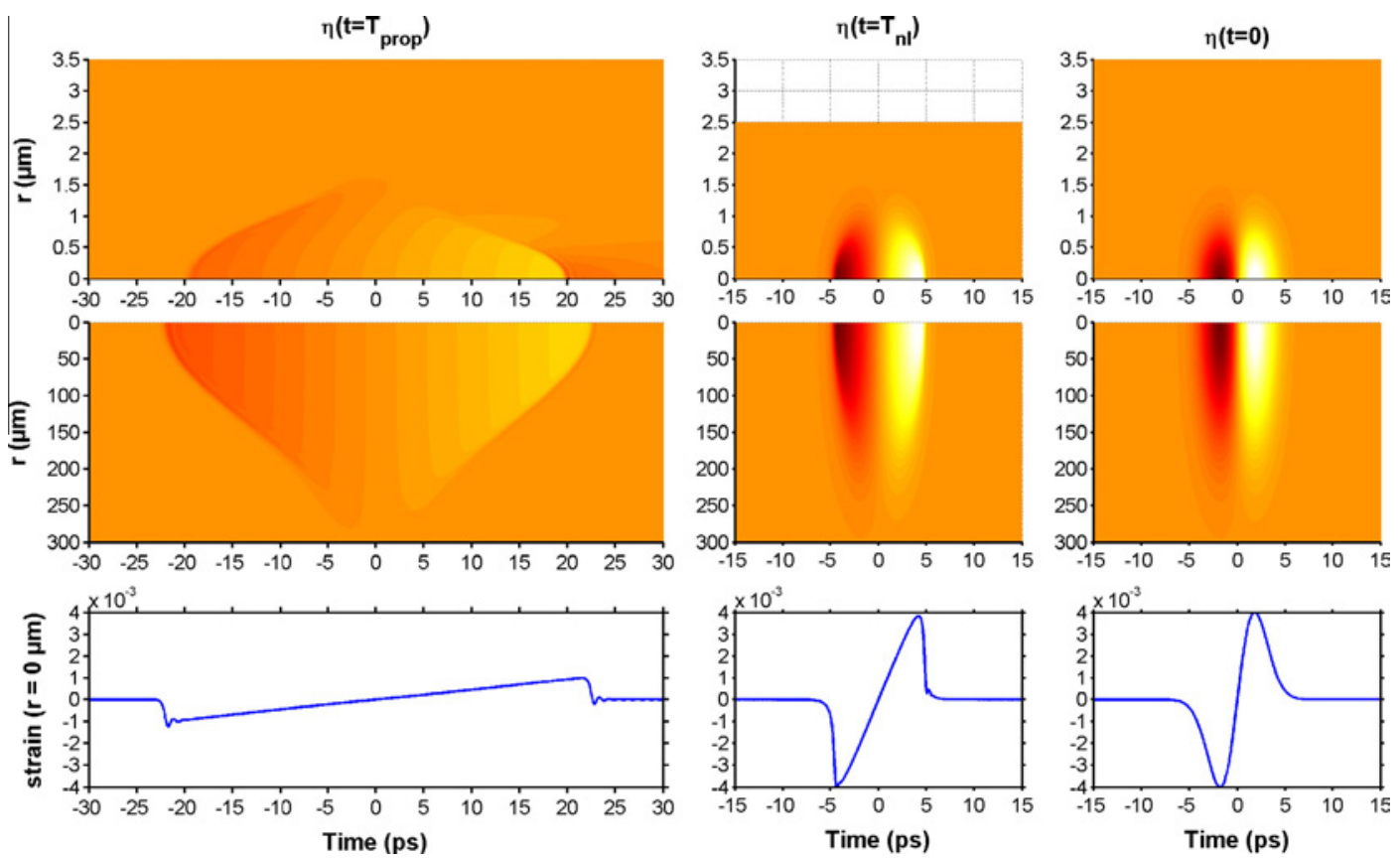

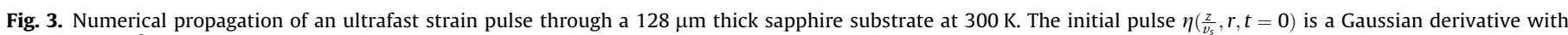

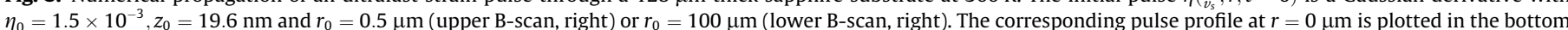

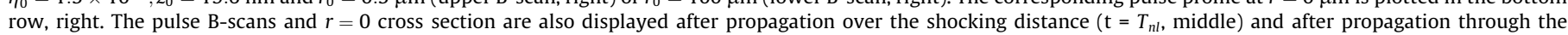
substrate $\left(\mathrm{t}=T_{\text {prop }}\right.$, left $)$.

transform, an alternative approach consists of using a discrete Hankel transform (dHT) scheme. ${ }^{2}$ For small enough time step, the FD scheme possesses better relative error than dHT algorithms; on the other hand dHT is unconditionally stable regardless the

\footnotetext{
${ }^{2}$ The Hankel transform is defined as $g(s)=2 \pi \int_{0}^{+\infty} f(r) J_{0}(2 \pi s r) r d r$.
}

time step. This allows to base the time step of the whole algorithm on the stability criteria of the CRK43 algorithm. Moreover, the discretizations along the depth direction $z$ (subscript $i$ ) and lateral direction $r$ (subscript $j$ ) are decoupled because the CRK43 algorithm operates along $z$ for fixed $r$, while the dHT algorithm operates along $r$ for fixed $z$. 
In order to take into account all the effect numerically, the CRK43 algorithm is used to treat all effects but diffraction, and the dHT is used to treat diffraction separately. Both algorithms are then used alternately according to the Strang splitting algorithm:

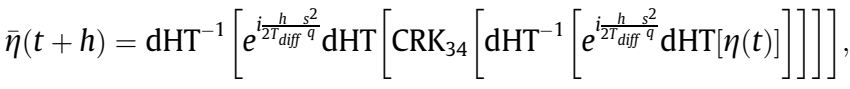

where $\bar{\eta}(t)$ is a matrix whose element is given by the value of the strain $\eta_{i j}(t)=\eta\left(z_{i}, r_{j}, t\right)$. The time step $h$ is chosen such that $h \ll \frac{z_{i+1}-z_{i}}{v_{s}}$. This numerical approach is valid as long as the sampling step $\left(z_{i+1}-z_{i}\right)$ stays smaller than the width of the shock front which may appear during the propagation. Actually, most of the ultrafast acoustics experiments in solids can be computed following this numerical scheme since acoustic dispersion and/or attenuation keep the shock slope to low enough value.

To illustrate the main features of nonlinear propagation, highamplitude, short acoustic pulses are mapped before and after propagation through GaAs at $20 \mathrm{~K}$ (see Fig. 2) and sapphire at room temperature (see Fig. 3). In both cases, the computation has been performed for two different pulse radii $\left(r_{0}\right)$ such that diffraction effects are significant (smaller radius, upper B-scans) or not (larger radius, lower B-scans).

The choice for the initial profile has been deduced from experimental results (see Section 3 ) such that $\eta(z, r, t=0)=$ $\eta_{0} e^{\frac{1}{2} \frac{z}{\delta}} \mathrm{e}^{-\frac{1}{4}\left(\frac{r}{r_{0}}\right)^{2}} e^{-\frac{1}{2}\left(\frac{z}{\delta}\right)^{2}}$ with $z_{0}=\frac{e \delta}{2}$.

When the initial pulse strain is large enough, nonlinear behavior dominates such that the short pulse evolves into a $\mathrm{N}$-wave (i.e. shock wave) in both cases (see middle B-scans and plots in Figs. 2 and 3 at time $t=T_{n l}$ ).

While the attenuation can safely be neglected for $370 \mu \mathrm{m}$ thick GaAs at low temperatures, it cannot be neglected for sapphire $128 \mu \mathrm{m}$ thick at room temperature [28].

Whereas the strain pulse continues to evolve like a shock wave in sapphire at room temperature (see left B-scans and plot in Fig. 3 computed at $t=T_{\text {prop }}$ ), it evolves into a train of solitons followed by an oscillating tail in GaAs at low temperature (see left B-scans and plot in Fig. 2 computed at $t=T_{\text {prop }}$ ). The properties of this two different propagation regimes are the subject of Sections 5 and 6, respectively.

The dashed lines in the left B-scans in Fig. 2 emphasize the shape of the solitonic wave front with and without diffraction as discussed by Kawasaki et al. [47]. Starting with a planar wavefront, the soliton profiles follow the Gaussian intensity profile when diffraction is low (dotted line of lower left B-scans). The main effect of the diffraction is to radially spread the low frequency components of the nonlinear pulse, effectively reducing the nonlinear effect (which is strongest when all the frequency components are in phase) and induce a parabolic profile. Hence the soliton shape becomes parabolic in the strong diffraction regime; such a solution can be found by similarity but is not a soliton anymore. The trailing part of the nonlinear pulse is also sensitive to diffraction effects but the parabolic bending is now opposite to the Gaussian profile. It so happens that both effects cancel each other quite nicely around $\frac{T_{\text {prop }}}{2}$ in this case.

Starting with a planar wavefront, the soliton profiles become parabolic in the strong diffraction regime whereas they follow the Gaussian intensity profile when diffraction is low (dotted line of lower left B-scans).

\section{Propagation in the linear regime}

At low laser fluence, the nonlinear term may be dropped such that the propagation becomes linear and can be solved analytically.
Assuming a Gaussian transverse profile for the initial strain pulse and after taking a Fourier transform along $z$ and a Hankel transform along $r$, Eq. (5) provides the solution $\tilde{\eta}(q, r, t)$ such that

$\tilde{\eta}(q, r, t)=\frac{1}{1-i \frac{q_{d}}{q}} e^{-\frac{11}{2}{ }_{1-i \frac{q_{d}}{q}}^{2}} e^{-\frac{q^{2} t}{T_{\text {att }}}-i \frac{q^{3} t}{T_{\text {disp }}}} \tilde{\eta}(q, r=0, t=0)$,

where $\tilde{\eta}(q, r=0, t=0)$ is the initial pulse profile at the epicenter in the Fourier domain and $\tilde{\eta}(q, r, t)$ the pulse profile after a propagation time $t$. The diffraction effect is driven by the parameter $q_{d}=\frac{t}{2 \pi^{2} T_{\text {diff }}}=\frac{\xi^{2} c_{0} z_{0} t}{4 \pi^{2} r_{0}^{2}}$

Therefore, it is possible in the linear regime to retrieve the initial pulse analytically by measuring the acoustic pulse after propagation through the substrate, at least if the acoustic properties of the substrate are known. Alternatively, if both the initial pulse and the pulse after propagation are known, it is possible to determine the acoustic properties of the substrate. Moreover, in the linear regime effects of attenuation, dispersion and diffraction can be emphasized at will by tuning the experimental conditions.

\subsection{Dispersion}

A first example of tuning experimental conditions in the linear regime is shown in Fig. 4. In this experiment, the diffraction effect was small such that the change in the pulse profile is mainly driven by the acoustic dispersion. The experiment was performed at a temperature of $20 \mathrm{~K}$ to minimize attenuation. Thus, $T_{\text {disp }} \ll T_{\text {att }}, T_{\text {diff }}$.

A strain pulse has been generated by an excited aluminum layer and detected in another aluminum layer (both $40 \mathrm{~nm}$ thick) after propagation through $356 \mu \mathrm{m}$ of GaAs. The surface displacement $u_{\text {exp }}\left(z, r=0, t_{\mathrm{Al}}+t_{\mathrm{GaAs}}\right)$ is directly measured by an interferometer.

This displacement is the result of the original strain pulse and multiple reflections inside the Al layer. To retrieve the displacement $u_{\mathrm{GaAs}}\left(z, r=0, t_{\mathrm{GaAs}}\right)$ due to the pulse arriving at the $\mathrm{Al} / \mathrm{GaAs}$ interface, we use the transfer matrix formalism (see the review paper by Matsuda et al. in this issue). Eq. (7) can then be used to retrieve the initial pulse $u_{0}(z, r=0, t=0)$ before its propagation through the GaAs substrate using standard value for GaAs acoustic properties. In this case we find that the initial displacement can be fitted nicely by a Gaussian pulse profile, hereby motivating the use of Gaussian derivative as initial strain pulse in the numerical computation in Figs. 2 and 3.

Attenuation has been neglected during the propagation inversion because of the noise amplification effect. According to Eq. (7), viscous attenuation is equivalent to convolution with a Gaussian

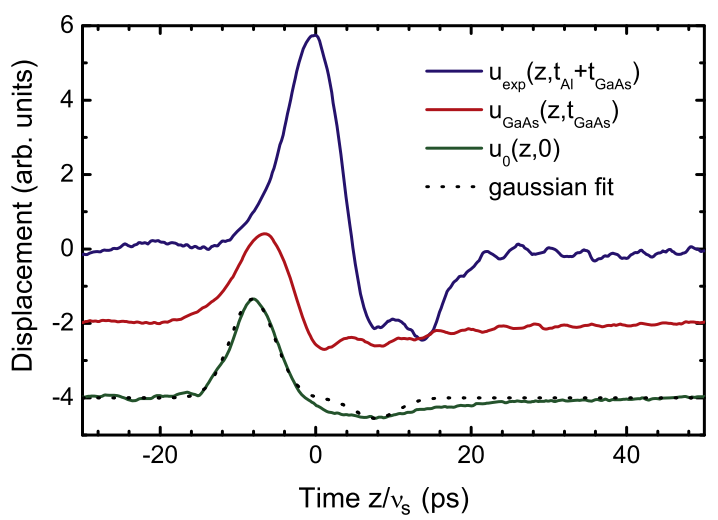

Fig. 4. Displacement pulses $u_{\text {exp }}\left(z, r=0, t_{\mathrm{Al}}+t_{\mathrm{GaAs}}\right)$ measured at sample surface (blue), $u_{\mathrm{GaAs}}\left(z, r=0, t_{\mathrm{GaAs}}\right)$ retrieved at $\mathrm{Al} / \mathrm{GaAs}$ interface (red) and $u_{0}(z, r=0, t=0)$ before propagation through the GaAs substrate (green). Dotted line: displacement of Gaussian initial pulse. (For interpretation of the references to colour in this figure legend, the reader is referred to the web version of this article.) 
function such that the fitted Gaussian pulse shape may be interpreted as a broadened initial strain pulse. Let us assume that the transducer is homogeneously strained (which is a fair assumption for a thin Al layer), yielding a rectangular initial strain pulse with a width equal to the Al layer thickness. By equating the full width at half maximum of this strain pulse convoluted with a Gaussian and the retrieved initial pulse, we find $\frac{\mu}{2 \rho}=3.6 \times 10^{-4} \mathrm{~nm}^{2} \mathrm{ps}^{-1}$ at $20 \mathrm{~K}$. It appears that this value is one order of magnitude too high to nicely fit the nonlinear experiment presented below. This is an indication that viscosity is not the perfect model for GaAs at low temperature. The discrepency may come from the fact that fits for nonlinear experiments are more sensitive to attenuation above $200 \mathrm{GHz}$, while for linear experiments they are more sensitive to attenuation below $200 \mathrm{GHz}$. Indeed, attenuation in crystals, especially at low temperature is not a trivial thing, as well explained in Ref. [49].

In contrast to attenuation, dispersion is well taken into account by our model Eq. (7). Hao and Maris have nicely demonstrated the acoustic dispersion in quartz, silicon, germanium, GaAs and sapphire by measuring the chirped subsonic oscillating tail of an acoustic pulse after propagation through several hundreds of micron [20]. The acoustic chirp may also be revealed by looking at the phase spectrum. We can assume that the spectral phase of the initial acoustic pulse is flat since all frequency components are generated simultaneously in the Al layer. Any phase shift in the pulse $u_{\mathrm{GaAs}}\left(z, r=0, t_{\mathrm{GaAs}}\right)$ must be due to the acoustic dispersion and diffraction.

The measured phase spectrum of $u_{\mathrm{GaAs}}\left(z, r=0, t_{\mathrm{GaAs}}\right)$ is plotted in Fig. 5. The fit function $\Phi\left(q, t=T_{\text {prop }}\right)$ is obtained by extracting the phase from Eq. (7) at $r=0$ :

$\Phi(q, r, t)=\gamma q^{3} t+\arctan \left(\frac{q_{d}}{q}\right)-\frac{1}{2} \frac{\frac{q_{d}}{q}}{1+\left(\frac{q_{d}}{q}\right)^{2}} \frac{r^{2}}{r_{0}^{2}}$

The fit function contains two coefficients: the Gaussian radius $r_{0}$ of the initial pulse which mainly determines the low-frequency phase behavior, and the dispersion coefficient $F$ determining high-frequency behavior.

We find that the phase is nicely reproduced by a cubic law as expected from the dispersion term in Eq. (7). Moreover, the fitted dispersion parameter $7.4 \times 10^{-3} \mathrm{~nm}^{3} \mathrm{ps}^{-1}$ is very consistent with a dispersion coefficient $\frac{F}{2 \rho v_{s}}=(7.4 \pm 0.3) \times 10^{-3} \mathrm{~nm}^{3} \mathrm{ps}^{-1}$ measured by Hao and Maris. [20]. The radius $r_{0}$ is fitted around $7 \mu \mathrm{m}$ but with limited confidence because of the low spectral resolution (i.e. the length of the pump-probe trace). We can only conclude that the diffraction effect is small in this experiment.

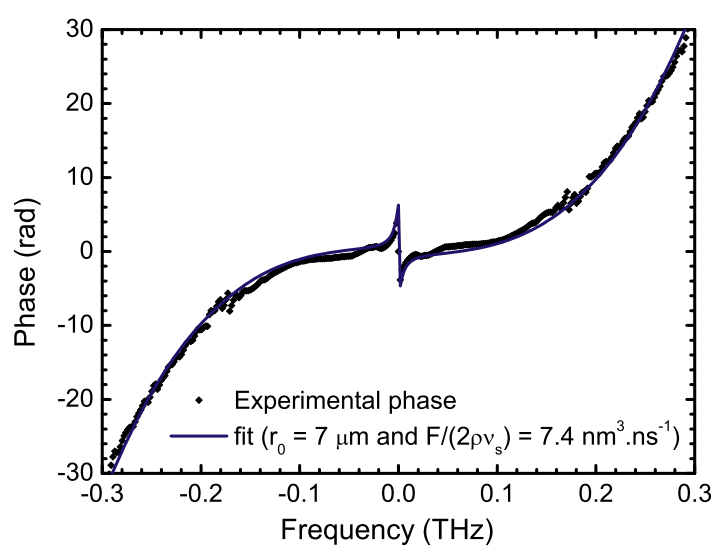

Fig. 5. Phase of the acoustic pulse after propagation through $356 \mu \mathrm{m}$ of GaAs (black diamond). The dotted line is the best fit with a cubic law according to Eq. (8).

\subsection{Diffraction}

Diffraction of ultrashort acoustic pulses is better revealed by tightly focusing the pump beam. It has been nicely demonstrated by Daly and coworkers who used microscope objectives for the pump and probe beams [50]. They measured and fitted the distortion of the pulse shape after propagation though a silicon substrate. By inspecting Eq. (7), one can see that the pulse shape at $r=0$ looks like its derivative in the far fields $q_{d} / q \gg 1$ (dispersion and attenuation put aside).

This shape change can be exploited to reconstruct the wave front of the initial strain pulse once the wavefront is measured in the far field. Using a Fresnel integral Daly et al. were able to reconstruct the transverse spatial profile of pulses generated by an 1D array of $\mathrm{Al}$ stripes and a 2D array $\mathrm{Al}$ dots with a lateral resolution of the order of at most $400 \mathrm{~nm}$ [50].

Interestingly, when the pump pulses are tightly focused, strain pulses with transverse polarization are also generated and propagate through the substrate (see Ref. [35] for an example in GaAs). However, in contrast with longitudinal waves, it is not possible in most cases to propagate back the transverse wavefronts because of the elastic anisotropy which give rise to cusp singularities in the wavefront. Therefore a direct approach is necessary as the one developed by Every et al. based on the Green function formalism [51]. Combining longitudinal wavefront inversion and direct transverse wavefront reconstruction should lead to better resolution in ultrashort acoustic imaging experiments.

\section{Propagation in the nonlinear regime}

The effects of nonlinear propagation at higher pump fluence are illustrated in Fig. 6, which shows the ultrashort strain pulse after propagation through a GaAs substrate in the linear (lower scans) and nonlinear regime (upper scans).

The nonlinearity is evidenced by two specific features. It appears that the nonlinear wavefront propagates faster than the sound velocity which is a typical signature of nonlinear effects. The closer the signal is to the epicenter $r=0 \mu \mathrm{m}$, the stronger the nonlinearity affects the pulse shape. A closer look around the epicenter shows an oscillation lasting much longer in the nonlinear case. Moreover, the nonlinear signal spreads much further away from the epicenter while initial pulse profiles are identical. Fig. 6 is a good example of the nonlinear propagation of ultrashort pulses and compares nicely with the computation shown in Fig. 2. Like in the computations, a train of short pulses with parabolic shape appears at the front of nonlinear wave. Let us focus on the signal recorded at $r=0 \mu \mathrm{m}$ where the nonlinearities are strongest. Fig. 7(a) displays an interferometer signal at $r=0 \mu \mathrm{m}$ for three different pump intensities. At the lowest intensity the propagation is linear and the pulse shape is very similar to what Daly et al. have measured in Si [50]; it can be computed with Eq. (7). When increasing the pump intensity the pulse broadens and sharp features appear at the front. At the highest pump intensity, the trailing oscillations due to acoustic dispersion are clearly visible. Moreover, the front of the pulse (negative time delay) is stiffening while the amplitude of the pulse increases, resembling the stiffening of wavefront during a shock formation.

When looking at Fig. 7(b) the broadening of the acoustic spectrum is clear. While the frequency range hardly reaches $0.3 \mathrm{THz}$ for the linear pulse, it goes up to $0.8 \mathrm{THz}$ in the nonlinear regime. The limit of the detected bandwidth is to some extent experimental. The sensitivity at a particular high frequency has been dramatically enhanced in sapphire (ruby) taking advantage of a $0.83 \mathrm{THz}$ resonant electronic two-level system [52]. 


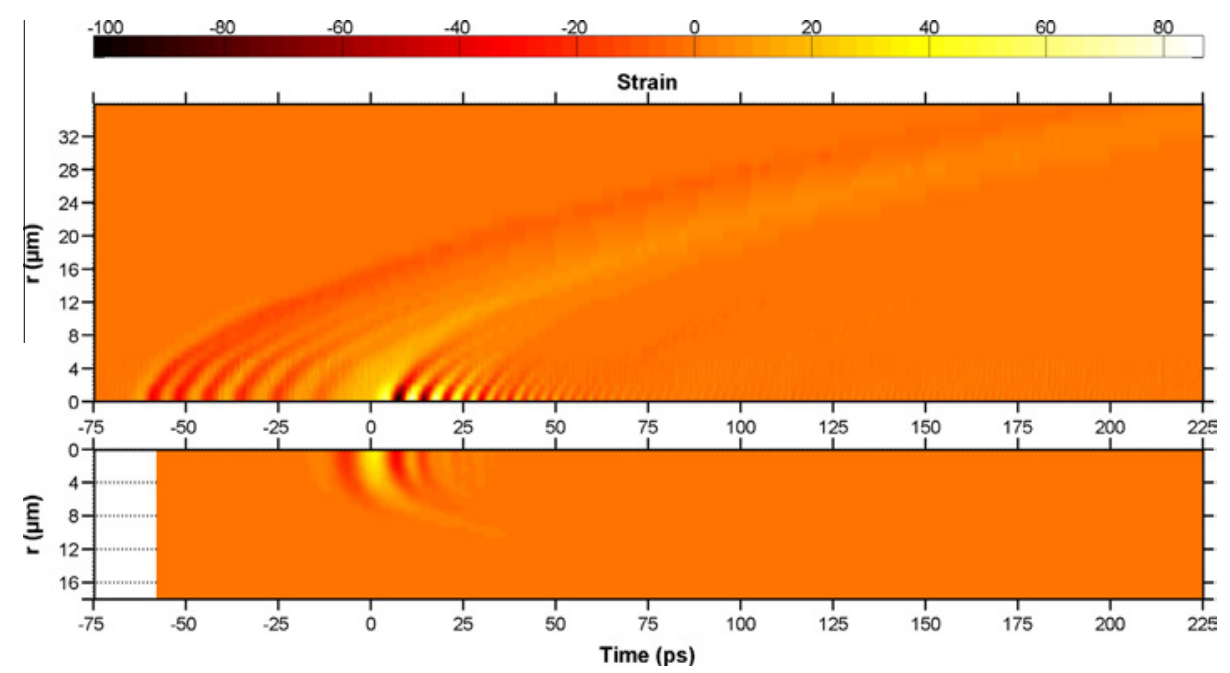

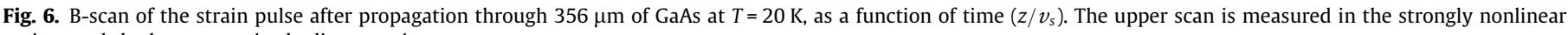
regime and the lower scan in the linear regime.

(a)
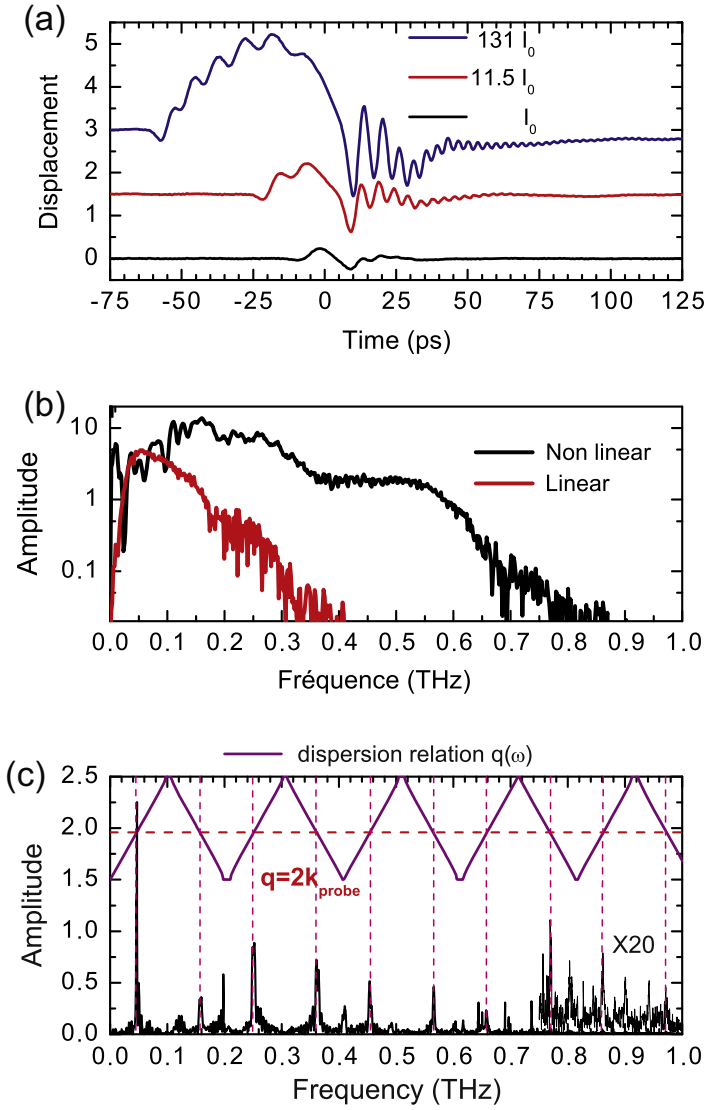

Fig. 7. (a) Displacement as a function of time $\left(z / v_{s}\right)$ measured at $T=20 \mathrm{~K}$ and at $r=0 \mu \mathrm{m}$ in the linear, weakly nonlinear and strongly nonlinear regime (respectively $I_{0}, 11 I_{0}$ and $131 I_{0}$ ) at a pump beam size $r_{0}=2.1 \mu \mathrm{m}$. (b) Spectral amplitude in linear (red) and strongly nonlinear (black) case. (c) Spectral amplitude of nonlinear wave when detected by a superlattice (SL). The dispersion relation of the SL is plotted to retrieve the Brillouin peaks of high sensitivity. (For interpretation of the references to colour in this figure legend, the reader is referred to the web version of this article.)

For GaAs the sensitivity at high frequencies can be boosted by measuring the reflectivity of a superlattice instead of using a thin Al layer. This is caused by resonance between the optical wave vector $k_{\text {probe }}$ and acoustic modes $q=2 k_{\text {probe }}$ of the superlattice (see Ref. [53] for more details). The dispersion of the acoustic mode is plotted at the top of Fig. 7(c). Thanks to the increase of sensitivity at the resonant frequencies $q$ it becomes evident that the nonlinear pulse spectrum extends up to $1 \mathrm{THz}$ at least.

It is noteworthy that nonlinearity enhances the low-frequency part of the spectrum $(<0.05 \mathrm{THz})$ compared to the linear spectrum. Since the low frequencies are diffracting more than higher ones during propagation, it explains why the diffraction wings observed in Fig. 6 are more pronounced in the nonlinear case as compared to the linear case.

The initial pulse profile at low laser fluence must differ from that at high fluence since the photothermal generation process is nonlinear as well. However the difference is expected to be small for thin aluminum films since the pulse shape is strongly constrained by the film thickness.

As was mentioned in the Introduction, one of the most fascinating aspects of nonlinear ultrasonics is the formation of acoustic solitons. Solitons will be discussed in more detail in Section 6 . Nonlinear diffraction has significant effects on the stability of solitons [44,24]. The results in Figs. 2 and 6 imply that true solitons are unlikely to appear in ultrashort acoustic experiments with finite pump spots as long as diffraction occurs, even in absence of attenuation. The sharp features in Fig. 6 propagating faster than the sound are soliton-like features but no real solitons.

Fortunately, diffraction can be overcome in most experiments. In the example in Fig. 6, the pump beam is focused with the help of an asymmetric molded lens down to $r_{0}=0.7 \mu \mathrm{m}$. When the pump is much less focused (e.g. $r_{0}=5 \mu \mathrm{m}$ ), the associated diffraction time $T_{\text {diff }}$ becomes longer than the time $T_{\text {prop }}$ it takes for the pulse to travel through $356 \mu \mathrm{m}$ of GaAs. As the computations in 2 (center line, left) show, in that case the lateral supersonic soliton velocity follows the Gaussian intensity profile.

Practically, diffraction can then be ignored and this allow us to further simplify Eq. (5) to the Korteweg-de Vries-Burgers equation

$\partial_{t} \eta=\frac{T_{\text {prop }}}{T_{n l}} \eta \partial_{z} \eta-\frac{T_{\text {prop }}}{T_{\text {disp }}} \partial_{z z z}^{3} \eta+\frac{T_{\text {prop }}}{T_{\text {att }}} \partial_{z z}^{2} \eta$.

Depending on the experimental conditions, this equation can be further simplified and applies to two very interesting cases of nonlinear ultrafast acoustics experiments: ultrafast shock wave as demonstrated by van Capel et al. $[18,19]$ and real soliton waves as first demonstrated by Hao and Maris [20]. Both wave types are solutions of two classic differential equations which are presented in Section 5 and 6 with their specific properties. 


\section{Ultrafast shock waves - Burgers equation}

At room temperature, the dispersion term in Eq. (7) can be neglected, since attenuation quickly damps out high frequencies in the wave packet. Under the additional assumption of onedimensional propagation, Eq. (7) reduces to

$\partial_{t} \eta=-\frac{C_{N L}}{2 v_{s} \rho} \eta \partial_{z} \eta+v \partial_{z z} \eta$

with $v=\mu /(2 \rho)$. This equation can be solved numerically so that $\eta(z, t)$ can be obtained for each propagation time $t$ [18]. A more elegant approach is possible. By defining a scaled function $c(z, t)=\frac{C_{N L}}{2 v_{s} \rho} \eta(z, t)$ we find the Burgers equation

$\partial_{t} c=-c \partial_{z} c+v \partial_{z z} c$.

Applying the nonlinear Cole-Hopf transformation $c=-v \phi_{z} / \phi$ redefines the problem in the heat equation [39]:

$\partial_{t} \phi=v \partial_{z z} \phi$.

The solution of this equation for an arbitrary initial pulse $\phi(z, t=0)$ can be found by calculating its convolution with the Green's function solution $\Phi(z, t)=1 / \sqrt{4 \pi v t} \exp \left(-z^{2} / 4 v t\right)$ of Eq. (12):

$\phi(z, t)=\phi(z, t=0) * \Phi(z, t)$

This means that for each known initial pulse profile, the profile after propagation time $t$ can be calculated. For the empirical Gaussian derivative pulse, no analytical expression can be obtained for $\phi(z, t)$ and $\eta(z, t)$. It is however possible to perform a numerical convolution.

In order to observe nonlinear effects, nonlinearity must dominate attenuation. Whitham [39] defines an initial Reynolds number $R_{0}=\int_{0}^{\infty} c(z, t=0) \mathrm{d} z$ for a general symmetric bipolar pulse. Above $R_{0} \sim 1$, we can expect to observe nonlinear effects. When $R_{0} \ll 1$, the pulse will propagate linear, being continuously damped.

For a Gaussian derivative we calculate $R_{0}=\frac{C_{N L} z_{0}}{2 \sqrt{\sqrt{2} \mu \nu_{s}}} \eta_{0}$. For a pulse in sapphire at room temperature $\mu /(2 \rho)=6.3 \times 10^{-2} \mathrm{~nm}^{2} / \mathrm{ps}$, $C_{N L}=-18.5 \times 10^{11} \mathrm{~N} / \mathrm{m}^{2}, \mathrm{z}_{0}=38 \mathrm{~nm}$; this means that we might expect nonlinear behavior above a feasible $\eta_{0} \sim 3.3 \times 10^{-4}$. For our $126 \mu \mathrm{m}$ thick sample and this strain amplitude this gives $T_{\text {att }}=23 \mathrm{~ns}>T_{\text {prop }}=11.2 \mathrm{~ns}>T_{n l}=8.3 \mathrm{~ns}$, so that the calculated $R_{0}$ is in line with our analysis in Section 2. Note that insulators such as sapphire have low intrinsic damping, necessary for a large attenuation time and high $R_{0}$ [54].

The typical wave shape found after propagation in case of a high $R_{0}$ is the N- or shock wave, Fig. 8(b). Here, the steepness of the front and rear is determined by both the initial strain amplitude $\eta_{0}$ and the attenuation $v$.
The following series of measurements illustrates nonlinear propagation for a sapphire slab of $126 \mu \mathrm{m}$ thick (propagation time $11.22 \mathrm{~ns}$ ). The $\mathrm{Cr}$ generation and detection films were respectively $200 \mathrm{~nm}$ and $30 \mathrm{~nm}$ thick. The width of the pump beam was $r_{0}=58 \mu \mathrm{m}$. The calculations in Fig. 3 suggest that diffraction is sufficiently small to allow for a 1D approach. The pump fluence varied from 1.2 to $26.4 \mathrm{~mJ} / \mathrm{cm}^{2}$.

Fig. 8(a) shows the detected interferometric phase signals $\delta \varphi$ after propagation. For the highest fluence, the $1 \%$ phase signal change corresponds to a surface displacement as high as $0.6 \mathrm{~nm}$. The most prominent effect is the strong pulse broadening, indicative of supersonic propagation for the compressive part(subsonic for tensile); the signal shape remains symmetric.

Fig. 8(b) shows the measurement result for the highest fluence. Here, we also show the calculated phase signal based on a numerical simulation of propagation using a Gaussian derivative input wave of width $z_{0}=48.7 \mathrm{~nm}$ and amplitude $\eta_{0}=3.65 \times 10^{-3}$. The calculated strain profiles before and after propagation are shown in the inset in Fig. 8(b). The phase signal contains both a bulk metal contribution $\delta \varphi_{\text {metal }}$ and a surface displacement contribution $\delta \varphi_{\text {surface }}$. The former improves the fit in the form of the positive contributions at the front and rear of the main signal, when the strain at the surface is rapidly changing with respect to the travel time through the penetration depth, i.e. at the shock wave edges.

In first approximation, however, we may assume that the surface displacement contribution to the phase signal is dominant, so that the strain is proportional to the derivative of the phase signal. Although details in the signal are not represented accurately in this way, it does allow us to illustrate strain reshaping due to nonlinearity directly. The strain waveforms obtained in this way are shown in Fig. 9, right panels. All strain signals have been low-pass frequency filtered to reduce noise.

The sample was covered only half by a detection film so that we could perform measurements at the $\mathrm{Cr}$-sapphire interface of the generator film. This allows for a direct measurement of the input strain in sapphire. The left panels in Fig. 9 show the measured strain shapes upon entry in sapphire. The strains are fitted by a Gaussian derivative (solid lines), where we have allowed for different strain amplitude and width for the compressive and tensile parts. The excellent agreement indicates that $\phi(z, t=0)$ in Eq. (13) is known quite accurately.

The waveforms calculated via Eq. (13) for 11.22 ns propagation time are shown as solid lines in Fig. 9 in the right panel. Up to $18.8 \mathrm{~mJ} / \mathrm{cm}^{2}$, correspondence is quite accurate. The highest fluence of $21.1 \mathrm{~mJ} / \mathrm{cm}^{2}$ approaches the damage threshold and it might be that structural changes in the film induce strongly nonlinear or instable strain generation and decreased correspondence between theory and measurement. However, since the input strain still (a)

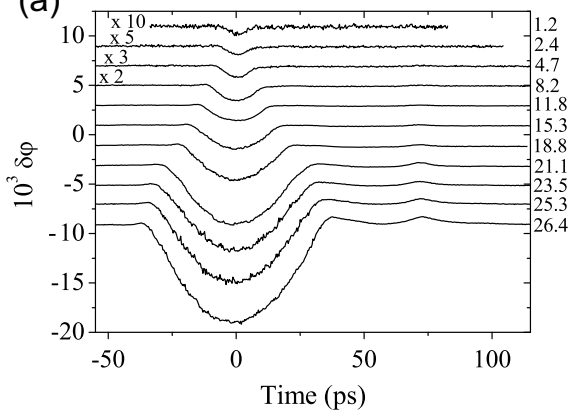

(b)

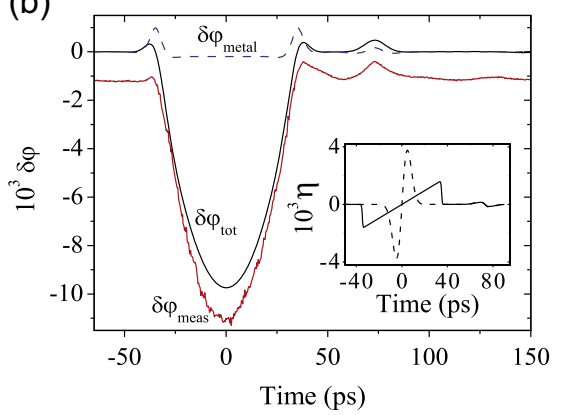

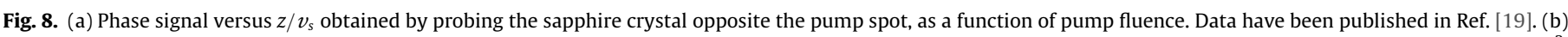

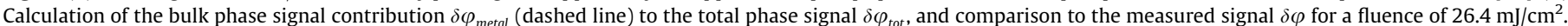

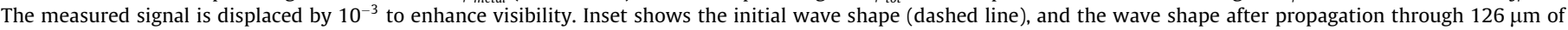
sapphire (solid line), obtained from a numerical calculation. 

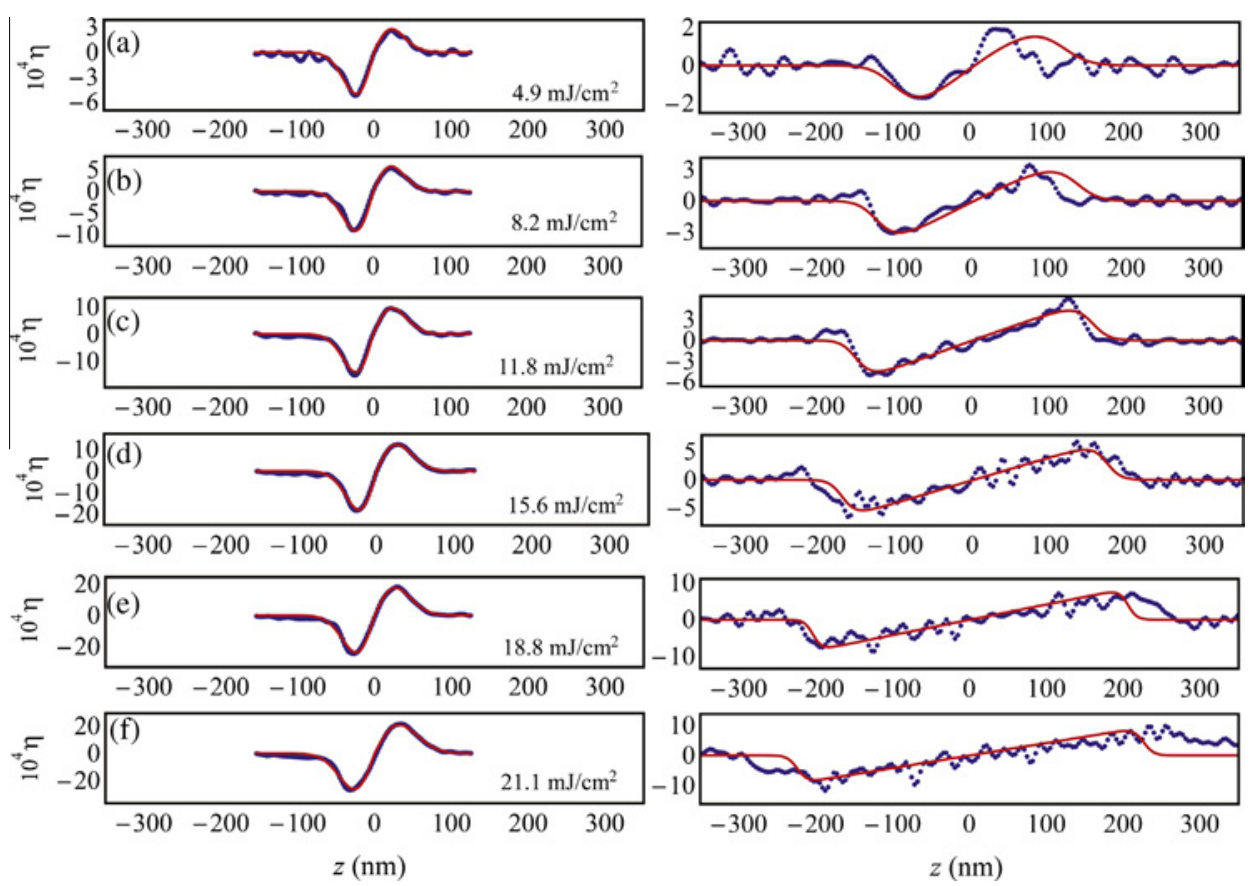

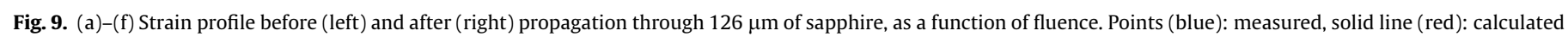

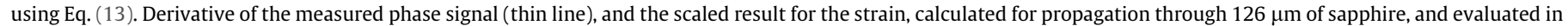
the metal film (thick line). (For interpretation of the references to colour in this figure legend, the reader is referred to the web version of this article.)

looks quite tractable, decreased correspondence could also point to additional nonlinear propagation effects not taken into account in the model. Further investigations in this amplitude regime could bring clarification.

At the lowest fluence (for which the initial strain amplitude is at the threshold of nonlinear behavior), we observe a broadening of the temporal profile after sample traversal, due to attenuation in the sapphire substrate. A calculation shows that the $1 / e$ attenuation for this sample thickness is at $67 \mathrm{GHz}$, a frequency near the $\sim 40 \mathrm{GHz}$ central frequency of the initial wavepacket. Thus, attenuation cannot be ignored even at the lowest fluences.

At the higher fluences, wave stretching and front steepening become apparent. The wavefronts seem to have equal steepness over the full nonlinear range. This is due to the fact that over this propagation distance, the steepness is fully determined by attenuation. The $10-90 \%$ rise time is determined at around $2.0 \mathrm{ps}$. When searching for a higher strain rate, one should use thinner samples. An example calculation shows that for a pump fluence of $18.9 \mathrm{~mJ} /$ $\mathrm{cm}^{2}$, a strain of $1.5 \times 10^{-3}$, or a pressure of $7 \mathrm{kbar}$, builds up in less than a picosecond for a 20 micrometer thick sapphire sample. A possible example of application is the study of material deformation under ultrafast shock compression [55].

When looking at subpicosecond phenomena, the time resolution of detection becomes increasingly important. The ultimate resolution of the interferometric measurements is limited by the largest of the probe pulse length, the surface roughness, and the parallelism of the sample planes. We determined the latter to be dominant: the angle between front and rear plane was determined at $\delta=0.076^{\circ}$, corresponding to an arrival time difference of $1.2 \mathrm{ps}$ over the $\sim 10 \mu \mathrm{m}$ probe spot.

Phonon attenuation in the detection film must also be taken into account. We may use a value of $150 \mathrm{GHz}^{-2} \mathrm{~m}^{-1}$ for attenuation in $\mathrm{Cr}$ [5], to estimate the $1 / e$ transmission frequency of the $30 \mathrm{~nm}$ detection film at $370 \mathrm{GHz}$. This would correspond to a temporal broadening around $2.7 \mathrm{ps}$.

At the highest fluences, we observe temporal smearing of up to $6 \mathrm{ps}$ at the shock wave edges. It is unlikely that additional broadening is due to diffraction or the variation of pump intensity over the probe spot (giving rise to arrival time differences of only $300 \mathrm{fs}$ ). We ascribe the nonlinear broadening due to laser intensity variations. Since the pump light is frequency-doubled to $400 \mathrm{~nm}$, the intensity noise in the pump is roughly twice the $\pm 1 \%$ of the amplified laser. We estimate that at a fluence of $26.4 \mathrm{~mJ} / \mathrm{cm}^{2}$, $\pm 2 \%$ in fluence corresponds roughly to a variation of $\pm 2.4 \mathrm{ps}$ in the shock front arrival times. The resulting $4.8 \mathrm{ps}$, in combination with the other sources of broadening, approaches the observed broadening of $6 \mathrm{ps}$.

Despite the fact that the phonon frequencies generated in this experiment are in the several hundreds of $\mathrm{GHz}$ range, the detection bandwidth is limited to at best $250 \mathrm{GHz}$. In Section 7, we discuss the increased demands on experiment design for high temporal resolution.

\section{Ultrashort acoustic solitons - Korteweg-de Vries equation}

When $T_{\text {att }}$ is large and the propagation time $T_{\text {prop }}$ increases such that it becomes comparable to the dispersion time $T_{\text {disp }}$, the shock description is not valid anymore. While the acoustic dispersion is not very sensitive to external parameters, the attenuation may be considerably decreased for most crystals by decreasing the lattice temperature. In fact, for standard substrates $(0.1 \mathrm{~mm}$ to $1 \mathrm{~mm}$ thick) and typical initial pulse profiles, there is a temperature range were $T_{\text {att }}$ becomes much larger than $T_{\text {disp }}$. The viscosity term in Eq. (9) can then be neglected. As an example, for a sapphire substrate of thickness $d_{\text {sample }}=300 \mu \mathrm{m}$, van Capel and Dijkhuis find $f_{\text {tr }}=1.3 \mathrm{THz}$ at $100 \mathrm{~K}, f_{\text {tr }}=6.5 \mathrm{THz}$ at $77 \mathrm{~K}$ and $>10 \mathrm{THz}$ at $60 \mathrm{~K}$ [16] where $f_{t r}$ is the frequency limit below which the attenuation is weak. Most ultrafast acoustic experiments are performed at low temperature (i.e. below liquid nitrogen temperature).

The so-called Korteweg-de Vries (KdV) equation is obtained once the diffraction and viscosity terms are dropped in Eq. (4). The typical development of strain pulses in the nonlinear, dispersive KdV regime is shown in Fig. 10. At least at the very beginning Fig. 10(b), the propagation scenario for the KdV equation is very 
similar to the Burgers equation. However, while the formation of a perfect shock is hindered by viscosity in the Burgers case, it is dispersion preventing shock formation in the KdV case. As was already observed in Fig. 7 but more directly in Fig. 10(d), the front part of the wave evolves into a train of solitons while the rear part of the pulse does not shock at all and shows oscillations.

It is the joint effect of nonlinearity and dispersion that leads to the formation of acoustic solitons [56-58]. The soliton is a general physical phenomenon that is found in for example fluid dynamics $[59,60]$, plasmas [61], optics [62-64] and atomic physics [65,66]. We will start with a theoretical discussion before presenting a more detailed analysis of experimental results.

\subsection{Solitons}

When diffraction and viscosity can be neglected, Eq. (4) can be rewritten into the Korteweg-de Vries (KdV) equation

$\partial_{t} \eta=-\sigma \eta \partial_{z} \eta-\partial_{z z z}^{3} \eta$

containing only one parameter $\sigma=-\frac{T_{\text {disp }}}{T_{n_{I}}}=\frac{C_{N L}}{F} \eta_{0} z_{0}^{2}$ when $t \rightarrow \frac{T_{\text {disp }}}{T_{\text {prop }}} t$. The KdV equation has been extensively studied, see Ref. [67], and possesses very intriguing properties. The KdV equation is very famous because of its particular solutions, special traveling waves named solitons. These solitons travel at constant speed and their shape stays identical during the propagation, even when encountering other solitons.

The soliton solutions of Eq. (14) are of the form (see Ref. [39])

$\eta_{s}(z, t)=\frac{12}{\sigma} k_{s}^{2} \cosh ^{-2}\left[k_{s}\left(z-4 k_{s}^{2} t-z_{s}\right)\right]$.

It is very important to note that, for a given $\sigma$ and initial strain profile, only one parameter $k_{s}>0$ determines all the soliton properties: amplitude $\frac{12}{\sigma} k_{s}^{2}$, speed in the comoving frame $4 k_{s}^{2}$, and inverse width $k_{s}$. Since most crystals have an acoustic dispersion coefficient $F>0$ and a nonlinear coefficient $C_{N L}<0, \sigma<0$ and solitons are generally compressive waves which travel faster than sound.

$z_{s}$ in Eq. (15) can be understood as an offset position at $t=0$ determined by the point of soliton formation. $k_{s}$ can be interpreted as the spectral width of the soliton as demonstrated by the Fourier transform $\tilde{\eta}_{s}(q, t)$ of Eq. (15):

$\tilde{\eta}(q, t)=\frac{6 \sqrt{2 \pi}}{\sigma} \frac{q}{\sinh \left(\frac{\pi q}{2 k_{s}}\right)} e^{-i q\left(4 k_{s}^{2} t+z_{s}\right)}$.

It is possible to predict the soliton properties from the initial shape of the strain pulse $\eta(z, t=0)$ prior to nonlinear propagation. The parameter $\sigma$ determining the soliton amplitude depends explicitly on $\eta_{0}$ and $z_{0}$.
The values of $k_{\mathrm{s}}$ which together with $\sigma$ define the soliton, can be determined from the initial pulse profile using an inverse scattering approach. There is an analogy between the parameter $k_{s}^{2}$ and the eigenvalues of bound states in a quantum potential well, where the quantum well profile resembles the initial compressive strain profile. Practically, one needs to find the eigenvalues $\lambda=-k_{s}^{2}$ of a stationary Schrödinger equation with a potential $V=-\frac{\sigma}{6} \eta(z, t=0)$. Anyone familiar with quantum mechanics will find this analogy very elucidating.

As previously discussed, $\sigma$ is negative for most materials. The existence of solitons therefore requires a negative strain and implies positive eigenvalues $k_{s}^{2}$. Hence, solitons are only observed as compressive waves generated from an initial compressive strain. Looking back to Eq. (15), positive eigenvalues mean that the speed of solitons is larger than the speed of sound and that the speed increases with the soliton amplitude.

Note that the analogy tells us that whatever the compressive strain, the number of solitons $N_{s}$ is at least one. Moreover the values of $N_{s}$ and $k_{s}^{2}$ can be evaluated analytically for some simple initial strain profiles. For example, when a thin layer is homogeneously strained by the pump laser, it is reasonable to assume a square profile for the compressive part of the strain [25]. In this case, $z_{0}$ is the layer thickness; $\left(N_{s}-1\right)^{2} \leqslant-\frac{\sigma}{6 \pi^{2}} \leqslant N_{s}^{2}$ provides the number of solitons and the $k_{s}$ values are given by the real solutions of $\tan ^{ \pm 1} \frac{\sqrt{-k_{s}^{2}-\frac{\sigma}{6}}}{2}=\mp \frac{k_{s}}{\sqrt{-k_{s}^{2}-\frac{\sigma}{6}}}$.

Interestingly, as $\eta_{0} \longrightarrow+\infty$ the largest $k_{s}^{2}$ parameter approaches $-\frac{\sigma}{6}$ such that the highest soliton amplitude is $2 \eta_{0}$, independent of crystal type. Taking a realistic initial strain amplitude $\eta_{0}=10^{-3}$ and width $z_{0}=20 \mathrm{~nm}$, solitons as large as $2 \times 10^{-3}$ and as short as $0.56 \mathrm{ps}, 0.41 \mathrm{ps}, 0.28 \mathrm{ps}$ and $0.14 \mathrm{ps}$ are expected in $\mathrm{GaAs}(100)$, $\mathrm{GaAs}(111), \mathrm{Al}_{2} \mathrm{O}_{3}(0001)$ and $\mathrm{MgO}(100)$ crystals respectively. The shortest soliton duration measured so far is $500 \mathrm{fs}$ in $\mathrm{Al}_{2} \mathrm{O}_{3}(0001)$ [28]. Therefore, the term femtosecond acoustics does not seem misplaced.

\subsection{Detection of soliton trains}

Before investigating the train of solitons in front of the pulse, let us focus on the trailing part of the pulse where acoustic dispersion is again clearly operative. The dispersive tail appears to last more than 300 ps. The dispersion is nicely revealed by applying successive band pass filtering every $0.1 \mathrm{THz}$ for example. The result is also shown in Fig. 11(a). The band pass filters are marked by the grid line slicing in the acoustic spectrum in Fig. 11(b), corresponding to the same strain pulse. In this case acoustic frequencies are

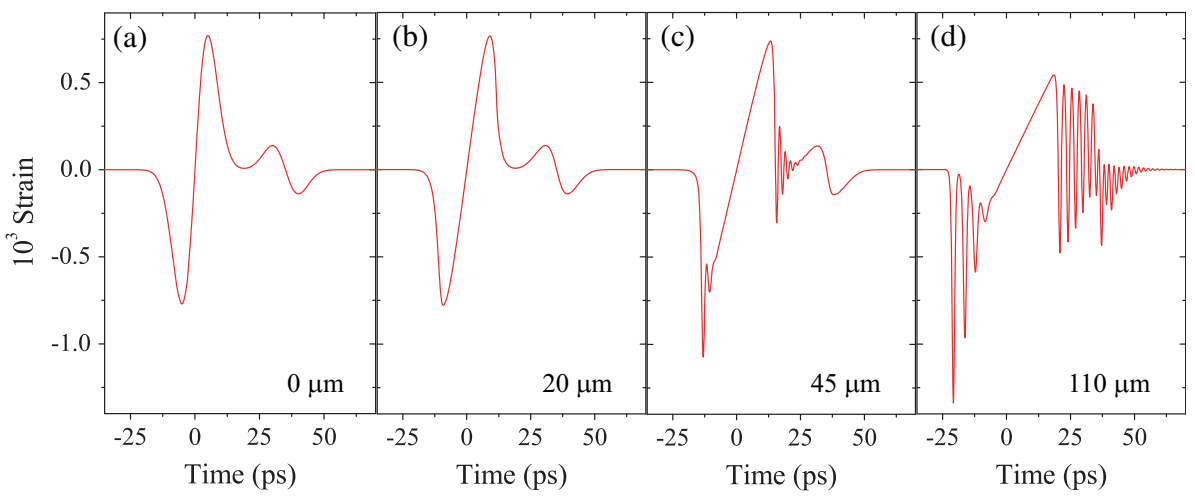

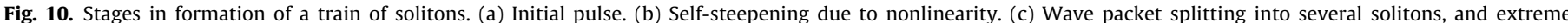

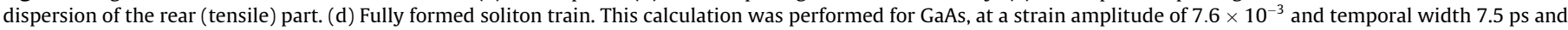
the indicated propagation distances. 
measured up to $0.85 \mathrm{THz}$. Like in the linear case, the phase displays a cubic dependence on frequency due to dispersion (see Fig. 11). The dispersion coefficient fit gives $7.1 \times 10^{-3} \mathrm{~nm}^{3} \mathrm{ps}^{-1}$, less than the value of $7.4 \times 10^{-3} \mathrm{~nm}^{3} \mathrm{ps}^{-1}$ found in the linear regime.

Such a discrepancy is expected since the high frequencies (above $0.3 \mathrm{THz}$ ) are not generated by the Al transducer but are generated progressively while the shock is forming. The correct value is retrieved when using a propagation distance of $345 \mu \mathrm{m}$ rather than $360 \mu \mathrm{m}$. The missing $15 \mu \mathrm{m}$ can be interpreted as a shock length, the length required to generate most high frequencies (cf. Fig. 10). According to the shock length definition $L_{\text {shock }}$ and using the pulse width $z_{0}=11.2 \mathrm{~nm}$ measured in the linear regime, the initial pulse amplitude $\eta_{0}$ can be evaluated around $9.44 \times 10^{-4}$, a reasonable value. This observation suggests that the propagation of high amplitude strain pulses in the KdV regime can be decomposed in two steps. First, the stiffest part of the strain pulse start to shock over a distance of the order of $L_{\text {shock}}$, creating all the high frequencies; then, the high frequencies propagate quasi-linearly.

The front part of the pulse is composed of a train of fast transient features of similar shape but different amplitude as expected for a train of solitons, see inset in Fig. 11(a). However the transient shape differs from the form Eq. (15) characteristic of KdV solitons because of generation and detection issues.

Since solitons are a product of a nonlinear effect, the waveform depends on the initial strain amplitude, i.e. the energy of the laser pulse heating the metallic layer. When averaging the signal over
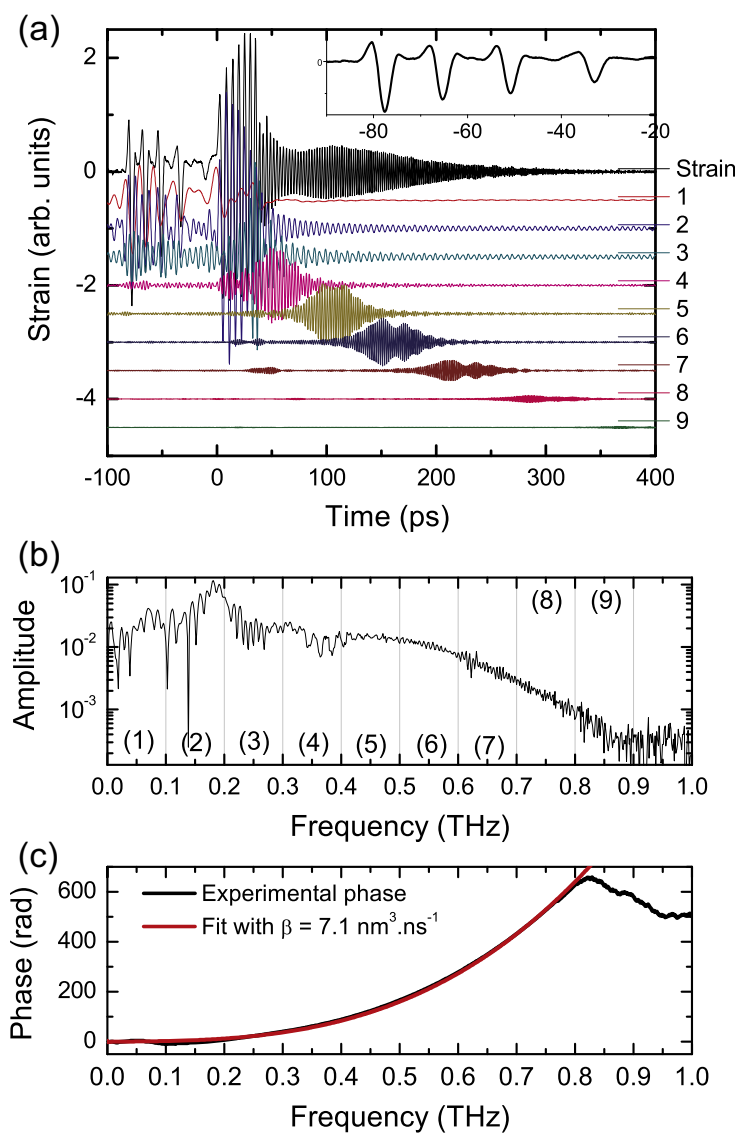

Fig. 11. (a) Black line: nonlinear strain pulse excited in $30 \mathrm{~nm}$ of aluminum with $12.5 \mathrm{~nJ}$ pulse energy, after propagation through $360 \mu \mathrm{m}$ of GaAs. The inset is a zoom of the soliton train. The signal is band pass filtered every $0.1 \mathrm{THz}$ (colored lines). The integer on right axis corresponds to the frequency band indicated in (b). (b) Spectral amplitude of the signal over the full spectral range. (c) Phase of the signal and the best cubic fit. (For interpretation of the references to colour in this figure legend, the reader is referred to the web version of this article.) many pulses for better signal-to-noise ratio, the power fluctuations of the pump laser induce a broadening of the soliton pulse as illustrated in Fig. 12(a). For a $\mathrm{kHz}$ amplified laser system the root mean square power fluctuation may be as high as $5 \%$, which largely explains the relative smoothness of ultrafast shock profile generated in sapphire (see also Section 5). For a Ti:sapphire oscillator the pulse-to-pulse energy fluctuation is usually less than $0.5 \%$ and insufficient to explain the discrepancy between theory and measurement for the results in Fig. 12.

However, as was already observed in Fig. 2, the nonlinearity also implies that the pulse profile varies over the radius $r$. When averaging the soliton profile over the probe laser spot, the profile is broadened as well but with an asymmetric shape, as illustrated in Fig. 12(b). When the ratio of the radii $r_{p}$ and $r_{s}$ of pump and probe respectively is close to or above one, the effect is dramatic. Even for $r_{s} / r_{p}=0.25$, as for the experiment in Fig. 11, the effect is very important.

The detection scheme also affects the shape of the observed soliton. Fig. 13(b) displays the complex relative change of reflectivity $\frac{\Delta r}{r}$ induced by a soliton as measured in a $30 \mathrm{~nm}$ Al layer. The soliton is detected through three different effects:

- the photo-elastic effect with a sensitivity resonant around the Brillouin frequency.

- the change in thickness of the thin film acting as a Fabry-Perot interferometer (i.e. the Fabry-Perot effect).

- the surface displacement of the sample which contributes only to the imaginary part.

All of them induce a dephasing of the probe pulse. The first two effects require the knowledge of the optical index and the photoelastic coefficient of the layer and substrate. Unfortunately, optical properties of thin films usually differ from that of the bulk. We attempt in Fig. 13(b) and (c) to model the soliton profile based on bulk value for optical index [68] $(n=2.4+8.6 i)$ or as determined by ellispometry $(n=0.9+5.3 i)$ by B. Gallas, INSP. In both
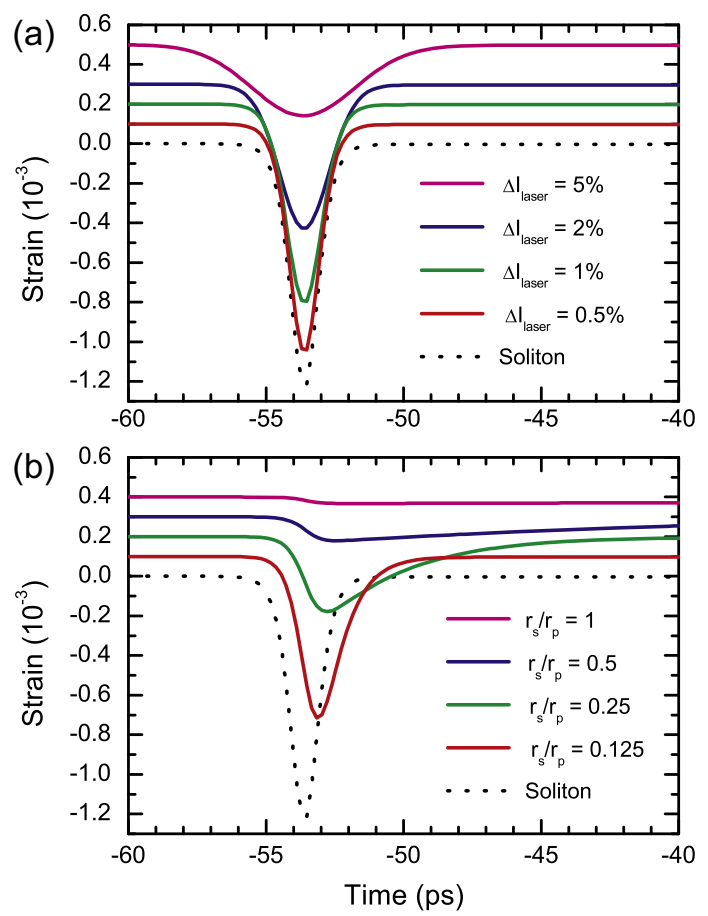

Fig. 12. (a) Effect of pump power fluctuation on the detected soliton for different relative variation. (b) Effect of the signal averaging by the probe transverse profile for different radius ratio. Curves are shifted vertically for clarity. 

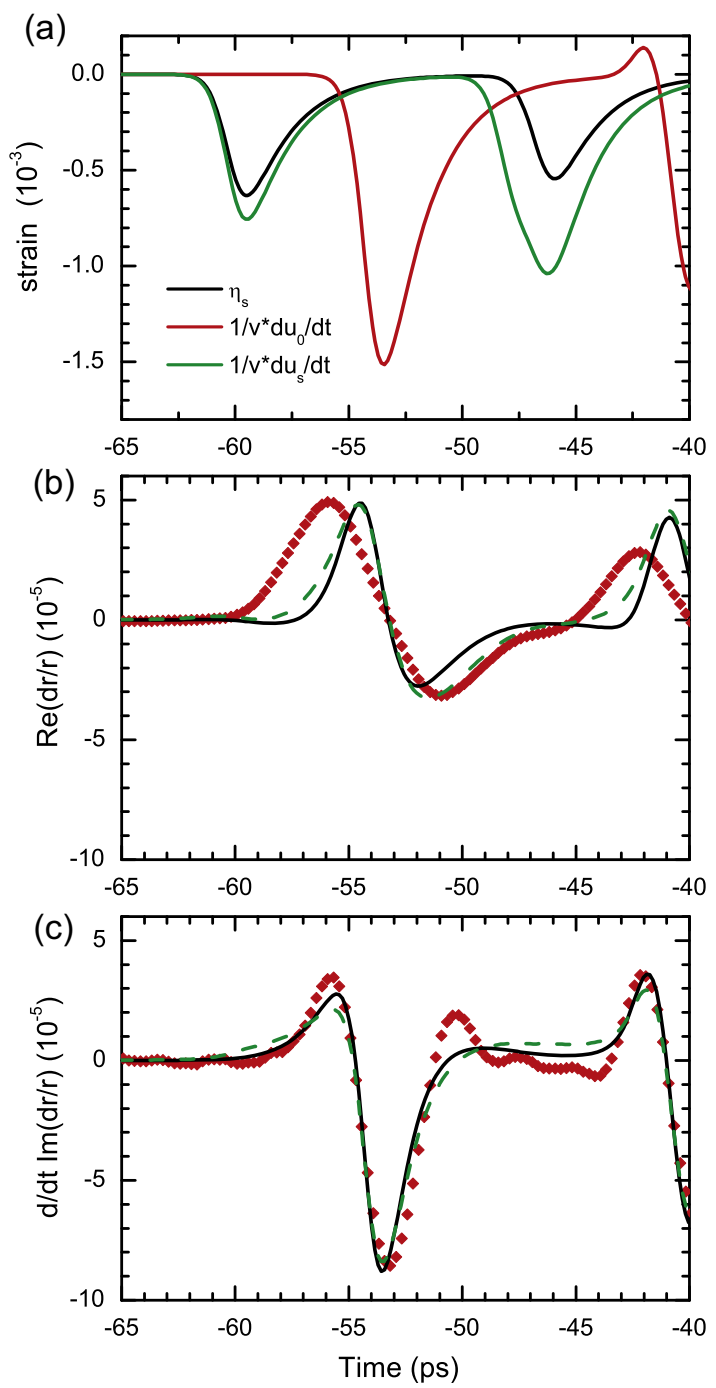

Fig. 13. (a) Incoming strain $\eta_{s}$ average by the probe profile (black). Derivatives of the surface $u_{0}$ and interface $u_{s}$ displacements are plotted in red and green respectively. (b) Real part of reflectivity variation measured (red diamonds) and computed using $\eta_{s}$ shown in (a). Two computation models are shown: one is based on the bulk Al optical index (green dash), the other on the effective optical index measured by ellipsometry (black line). (c) Idem for the imaginary part. (For interpretation of the references to colour in this figure legend, the reader is referred to the web version of this article.)

cases the photoelastic coefficient is the fit parameter. The thin film may also display surface/interface roughnesses which hinders the detection of high frequencies. ${ }^{3}$

\section{Discussion and outlook}

Various aspects (attenuation, diffraction, dispersion, nonlinearity) of long-distance nonlinear acoustic wave propagation have been quantitatively described, and correspondence to experimental results is excellent. The observations clearly prove that nonlinear, dispersive propagation in the form of shock waves and solitons is present.

\footnotetext{
3 The fit quality is slightly improved by also taking into account for the surface roughness which broaden the signal. The root mean square (RMS) surface roughness of the Al film probed in Fig. 13 has been estimated by atomic force microscopy (AFM) around $1.8 \mathrm{~nm}$ which leads to a small broadening of the signal (of the order of $0.29 \mathrm{ps})$
}

By virtue of the time resolution of subpicosecond acoustic experiments a precise measurement of the speed of the soliton gives direct access, via the equations of motion, to the amplitude and the width of the soliton. The fact that solitons are intrinsically stable over large propagation distances makes them interesting tools in nanometer manipulation and nondestructive testing. Since the formation length of solitons is only micrometers at the highest optical fluences, very compact sources of very frequency acoustic pulses may become available for high resolution acoustic microscopy.

Resonant interactions in combined photonic/phononic structures opens possibilities to strongly modify the material properties. First experiments showed the interplay between photonics and phononics at the nanoscale using acoustic solitons in nanocavities [69] and quantum wells [70].

Up to now there is no direct measurement of sub-picosecond solitons which are predicted by the theory, however. The quest for a femtosecond soliton is a very challenging one especially from a detection point of view. In the following, we address some current issues in femtosecond acoustics.

\subsection{Generation}

As has been shown in Section 2, the width and amplitude of the initial strain pulse are critical parameters to control nonlinear effects. One must use a stable source of femtosecond optical pulses with a probe radius much smaller than the pump radius (by a factor of ten at least). High strain amplitude may be generated with low repetition rate laser systems but the pulse-to-pulse fluctuation of intensity is a real issue when reaching the femtosecond acoustic regime (in contrast to high-repetition-rate systems presently available).

\subsection{Transducer}

Improved instrumental resolution also requires high-quality generation and detection layers. Especially in detection, the sample surface must be clean and flat with RMS roughness typically less than $1 \mathrm{~nm}$. Thin metallic films, like aluminum or chromium, are a good choice as transducer, yielding short strain pulses with large amplitude when excited by pulses of visible light. Indeed, when looking at the bulk metal properties, the penetration length (i.e. skin depth) varies between $10 \mathrm{~nm}$ to $100 \mathrm{~nm}$ typically and the strain amplitude can reach several times $10^{-3}$ when exciting just below the ablation threshold.

However, great care has to be taken when depositing the thin metallic film since optical, mechanical and thermal properties of thin films can differ greatly from the bulk ones. Much work has to be done in order to optimize the thin-film quality for a given substrate. The recent development in epitaxial growing techniques such as plasma enhanced atomic layer deposition provides highquality thin metal films, showing reduced scattering and attenuation. It would be then interesting to gradually alloy two different metals during the film growth, one chosen for its good adhesion and low impedence mismatch with the substrate and the other for its good optomechanical properties.

Whereas the conditions were met in previous experiments with sapphire [16] or MgO [25] substrates the pulse duration could only be retrieved by deconvolution with the apparatus sensitivity function.

However, the detection mechanisms can also be an issue in itself at such high frequencies. In particular, the assumption of a constant photoelastic coefficient is a quasistatic one: the electronic states are taken to follow the lattice vibrations adiabatically. This assumption has already been shown to have its limits in the detection of solitons with semiconductor multiple quantum wells [70]. Since the electron-phonon coupling constant in metals varies 
between several tens to a few hundreds of femtosecond in most metals, the quasistatic assumption should also be violated in thin metal film detection schemes at very high frequencies.

Note that this limitation applies to the detection of surface and interface displacements as well. Indeed the problem is arising from the fact that the probe light is not coupled directly to the lattice but senses the free electrons in the metal while the strain propagation is mainly related to bounded electrons. It is important to look for new detection schemes in order to improve the time resolution.

In this connection, the recent experiments based on X-rays $[71,72]$ or UV probe $[73,74]$ are promising. In both cases, the spatial resolution for wavefront detection can be improved as well. On the other side of the spectrum, one can use a $\mathrm{THz}$ detection scheme such as ultrafast antenna's in order to measure the electric field emitted by a thin dipolar layer strained by ultrashort strain pulses and connect this to the transient strain [75,76]. Another method is by probing strain via the piezospectroscopic effect [77]. Even more recent work explored the interaction between nonlinear shock wave and surface plasmons and connects the important field of plasmonics to ultrafast acoustics $[78,79]$.

\subsection{Acoustical beam shaping}

One direction that has not been explored in depth yet in nonlinear ultrafast acoustics is engineering of the optical femtosecond excitation profile in space and time. This can be achieved using spatial light modulators. Many interesting phenomena could arise. For example if one excites with a high power focused vortex beam (a diffraction limited ring) instead of a Gaussian shaped beam, one might create high-amplitude ring shaped acoustics wave fronts with interesting self focusing properties. Such focusing rapidly amplifies the acoustic gradients to a degree that the internal strength of the material is surpassed and acoustic ablation on the nanoscale takes place. Another example is to control the direction of travel of the high amplitude excited stain pulse and thus of the emergent solitons by properly sweeping the incident optical pulse in space and time. This scheme would allow the first study of colliding acoustic solitons.

\subsection{Imaging}

It has been shown that solitons exhibit nonlinear diffraction. When also taking into account effects in lateral dimensions, solitons appear to be highly diffractive, especially at impurities $[44,24,27]$. This nonlinear diffraction opens the way to wave-front reconstruction and nanometer-resolution fault detection [80] in the bulk of a material where electron microscopy is not possible.

Ultrafast acoustic waves may ultimately be used to perform acoustic 3D imaging at the nanoscale by measuring transmission and reflection at various angles. However, the emission angle resulting from diffraction depends mainly on the lateral size of the acoustic source, which is limited by the laser spot size. In the visible range it is very hard to get laser spots smaller than $400 \mathrm{~nm}$; ten times larger than the pulse length and almost one hundred times larger than the smallest wavelength found in ultrafast shock pulses. Hence high frequency acoustic waves barely diffract limiting the resolution of acoustic imaging to several hundreds of nanometers [50].

It would be interesting to obtain acoustic sources ten times smaller such that acoustic diffraction occurs for smaller acoustic wavelengths. In this regard, extension of ultrafast acoustics experiments in the XUV domain is again appealing, to improve the laser focusing and would open the road towards new transduction mechanisms and transducer types other than metallic films. Nonlinear excitation mechanisms such as multiphoton absorption may also offer new possibilities to realize nano-sources using non-metallic materials.

\subsection{Transverse ultrafast acoustics}

Up to now, only ultrashort longitudinal waves have been studied in the nonlinear regime. However intense high frequency transverse waves would be very useful as well to probe acoustic properties at the nanoscale. Whereas it is possible to generate transverse pulses from moderate to high intensity thanks to mode conversion [81-83] possibly combined with dilatation or acoustic anisotropy [84], nonlinear effects are barely cumulative for transverse waves in most crystals (whatever its orientation) which prevent the generation of transverse acoustic solitons. Propagation along the [111] axis in cubic crystals is one of the few candidates to provide cumulative nonlinearities for transverse waves and should be tested first.

\section{Conclusions}

Nonlinear ultrafast acoustics has become a mature field with potential for applications and new discoveries. Generation, propagation and detection of ultrafast acoustic pulses are understood in detail.

Ultrafast acoustics deals with very high frequency acoustic waves (THz) and short wavelengths (nanometers). Such waves suffer from severe damping in any material at reasonable temperatures. Therefore ultrafast acoustic methods are limited to but inherently suited for studying and manipulating small objects, i.e. nanostructures. Although controlled generation of high frequency acoustic waves is demonstrated in great detail, a sensitive detection of high frequency acoustic energy is not straightforward.

An acoustic CCD camera has unfortunately not been invented yet. However in bolometry, not subject of this paper, many advances take place that could make sensitive detection of short wavelength acoustic fields feasible and nondestructive acoustic testing on the nanoscale within reach.

Nonlinear ultrafast acoustics relies on the availability of stable, high power femtosecond lasers and suitable transducers. The tremendous developments both in ultrafast laser technology and fabrication of atomically flat transducers of any desired type will fuel further development and applications of ultrafast acoustics.

\section{References}

[1] C. Thomsen, H. Grahn, H. Maris, J. Tauc, Surface generation and detection of phonons by picosecond light pulses, Phys. Rev. B 34 (6) (1986) 4129, http:// dx.doi.org/10.1103/PhysRevB.34.4129.

[2] H. Maris, Picosecond ultrasonics, Sci. Am. 86 (1998) 278.

[3] C. Thomsen, J. Strait, Z. Vardeny, H. Maris, J. Tauc, J. Hauser, Coherent phonon generation and detection by picosecond light pulses, Phys. Rev. Lett. 53 (10) (1984) 989, http://dx.doi.org/10.1103/PhysRevLett.53.989.

[4] G. Eesley, Generation of nonequilibrium electron and lattice temperatures in copper by picosecond laser pulses, Phys. Rev. B 33 (4) (1986) 2144, http:// dx.doi.org/10.1103/PhysRevB.33.2144.

[5] T. Saito, O. Matsuda, O. Wright, Picosecond acoustic phonon pulse generation in nickel and chromium, Phys. Rev. B 67 (2003) 205421, http://dx.doi.org/ 10.1103/PhysRevB.67.205421.

[6] O. Wright, Ultrafast nonequilibrium stress generation in gold and silver, Phys. Rev. B 49 (14) (1994) 9985.

[7] K. Mizoguchi, M. Hase, S. Nakashima, M. Nakayama, Observation of coherent folded acoustic phonons propagating in a gaas/alas superlattice by two-color pump-probe spectroscopy, Phys. Rev. B 60 (1999) 8262-8266, http:// dx.doi.org/10.1103/PhysRevB.60.8262.

[8] M. Trigo, A. Bruchhausen, A. Fainstein, B. Jusserand, V. Thierry-Mieg, Confinement of acoustical vibrations in a semiconductor planar phonon cavity, Phys. Rev. Lett. 89 (2002) 227402, http://dx.doi.org/10.1103/ PhysRevLett.89.227402.

[9] N.-W. Pu, J. Bokor, Study of surface and bulk acoustic phonon excitations in superlattices using picosecond ultrasonics, Phys. Rev. Lett. 91 (2003) 076101.

[10] N. Stanton, R. Kini, A. Kent, M. Henini, D. Lehmann, Terahertz phonon optics in GaAs/AlAs superlattice structures, Phys. Rev. B 68 (11) (2003) 113302, http:// dx.doi.org/10.1103/PhysRevB.68.113302.

[11] C. Rossignol, B. Perrin, B. Bonello, P. Djemia, P. Moch, H. Hurdequint, Elastic properties of ultrathin permalloy/alumina multilayer films using picosecond 
ultrasonics and brillouin light scattering, Phys. Rev. B 70 (2004) 094102, http://dx.doi.org/10.1103/PhysRevB.70.094102.

[12] A.A. Maznev, K.J. Manke, K.-H. Lin, K.A. Nelson, C.-K. Sun, J.-I. Chyi, Broadband terahertz ultrasonic transducer based on a laser-driven piezoelectric semiconductor superlattice, Ultrasonics 52 (1) (2012) 1-4, http://dx.doi.org/ 10.1016/j.ultras.2011.07.007.

[13] O. Matsuda, T. Tachizaki, T. Fukui, J. Baumberg, O. Wright, Acoustic phonon generation and detection in $\mathrm{GaAs} / \mathrm{Al}_{0.3} \mathrm{Ga}_{0.7} \mathrm{As}$ quantum wells with picosecond laser pulses, Phys. Rev. B 71 (11) (2005) 115330. http://link.aps.org/abstract/ PRB/v71/e115330.

[14] K.-H. Lin, G.-W. Chern, Y.-K. Huang, C.-K. Sun, Terahertz electron distribution modulation in piezoelectric in $\mathrm{ga}_{1-\mathrm{x}} \mathrm{n} /$ gan multiple quantum wells using coherent acoustic nanowaves, Phys. Rev. B 70 (2004) 073307, http:// dx.doi.org/10.1103/PhysRevB.70.073307.

[15] C.-T. Yu, K.-H. Lin, C.-L. Hsieh, C.-C. Pan, J.-I. Chyi, C.-K. Sun, Generation of frequency-tunable nanoacoustic waves by optical coherent control, Appl. Phys. Lett. 87 (9) (2005) 093114. <http://link.aip.org/link/?APL/87/093114/1>.

[16] P. van Capel, D. Turchinovich, H. Porte, S. Lahmann, U. Rossow, A. Hangleiter, J. Dijkhuis, Correlated terahertz acoustic and electromagnetic emission in dynamically screened ingan/gan quantum wells, Phys. Rev. B 84 (8) (2011) 085317.

[17] E. Young, A. Akimov, R. Campion, A. Kent, V. Gusev, Picosecond strain pulses generated by a supersonically expanding electron-hole plasma in gaas, Phys. Rev. B 86 (2012) 155207, http://dx.doi.org/10.1103/PhysRevB.86.155207.

[18] P. van Capel, J. Dijkhuis, Optical generation and detection of shock waves in sapphire at room temperature, Appl. Phys. Lett. 88 (15) (2006) 151910. <http://link.aip.org/link/?APL/88/151910/1>

[19] P. van Capel, H. Porte, G. van der Star, J. Dijkhuis, Interferometric detection of acoustic shock waves, J. Phys.: Conf. Ser. 92 (2007) 012092, http://dx.doi.org/ 10.1088/1742-6596/92/1/012092.

[20] H.-Y. Hao, H. Maris, Experiments with acoustic solitons in crystalline solids, Phys. Rev. B 64 (2001) 064302, http://dx.doi.org/10.1103/PhysRevB.64. 064302.

[21] O. Muskens, A. Akimov, J. Dijkhuis, Coherent interactions of terahertz strain solitons and electronic two-level systems in photoexcited ruby, Phys. Rev. Lett. 92 (3) (2004) 335503. <http://link.aps.org/abstract/PRL/v92/e035503>.

[22] J. Maris, S. Tamura, Propagation of acoustic phonon solitons in nonmetallic crystals, Phys. Rev. B 84 (2) (2011) 024301.

[23] O. Muskens, J. Dijkhuis, High amplitude, ultrashort, longitudinal strain solitons in sapphire, Phys. Rev. Lett. 89 (28) (2002) 285504, http://dx.doi.org/10.1103/ PhysRevLett.89.285504.

[24] O. Muskens, J. Dijkhuis, High-amplitude, ultrashort strain solitons in solids, in: K.-T. Tsen (Ed.), Non-Equilibrium Dynamics of Semiconductors and Nanostructures, first ed., CRC Press, 2005, pp. 15-45.

[25] W. Singhsomroje, H. Maris, Generating and detecting phonon solitons in MgO using picosecond ultrasonics, Phys. Rev. B 69 (17) (2004) 174303. <http:// link.aps.org/abstract/PRB/v69/e174303>.

[26] B. Daly, T. Norris, J. Chen, J. Khurgin, Picosecond acoustic phonon pulse propagation in silicon, Phys. Rev. B 70 (21) (2004) 214307. <http://link.aps. org/abstract/PRB/v70/e214307>.

[27] E. Péronne, B. Perrin, Generation and detection of acoustic solitons in crystalline slabs by laser ultrasonics, Ultrasonics 44 (Suppl. 1) (2006) e1203.

[28] P. van Capel, J. Dijkhuis, Time-resolved interferometric detection of ultrashort strain solitons in sapphire, Phys. Rev. B 81 (14) (2010) 144106.

[29] A. Lomonosov, P. Hess, A. Mayer, Observation of solitary elastic surface pulses, Phys. Rev. Lett. 88 (7) (2002) 076104, http://dx.doi.org/10.1103/PhysRevLett. 88.076104.

[30] P. Hess, A. Lomonosov, Solitary surface acoustic waves and bulk solitons in nanosecond and picosecond laser ultrasonics, Ultrasonics 50 (2) (2010) 167.

[31] D. Funk, D. Moore, S. McGrane, J. Reho, R. Rabi, Ultra-fast spatial interferometry: a tool for characterizing material phase and hydrodynamic motion in laser-excited materials, Appl. Phys. A 81 (2005) 295, http:// dx.doi.org/10.1007/s00339-005-3214-4.

[32] J.-P. Monchalin, Optical detection of ultrasound at a distance using a confocal Fabry-Perot interferometer, Appl. Phys. Lett. 47 (1) (1985) 14.

[33] D. Hurley, O. Wright, Detection of ultrafast phenomena by use of a modified Sagnac interferometer, Opt. Lett. 24 (18) (1999) 1305.

[34] T. Tachizaki, T. Muroya, O. Matsuda, Y. Sugawara, D. Hurley, O. Wright, Scanning ultrafast Sagnac interferometry for imaging two-dimensional surface wave propagation, Rev. Sci. Instr. 77 (4) (2006) 043713. <http://link.aip.org/ link/?RSI/77/043713/1>.

[35] S. Zhang, E. Péronne, L. Belliard, S. Vincent, B. Perrin, Three-dimensional acoustic wavefront imaging in anisotropic systems by picosecond acoustics, J. Appl. Phys. 109 (3) (2011) 033507.

[36] F. Murnaghan, Finite Deformations of an Elastic Solid, John Wiley \& Sons, 1951.

[37] C. Kittel, Introduction to Solid State Physics, first ed., John Wiley \& Sons, 1996.

[38] N. Zabusky, M. Kruskal, Interaction of solitons in a collisionless plasma and the recurrence of initial states, Phys. Rev. Lett. 15 (1965) 240-243, http:// dx.doi.org/10.1103/PhysRevLett.15.240.

[39] G. Whitham, Linear and Nonlinear Waves, first ed., Wiley, 1974.

[40] K. Brugger, Thermodynamic definition of higher order elastic coefficients, Phys. Rev. 133 (1964) A1611-A1612, http://dx.doi.org/10.1103/PhysRev. 133.A1611.

[41] D. DiVincenzo, Dispersive corrections to continuum elastic theory in cubic crystals, Phys. Rev. B 34 (1986) 5450-5465, http://dx.doi.org/10.1103/ PhysRevB.34.5450.
42] B. Newberry, R. Thompson, A paraxial theory for the propagation of ultrasonic beams in anisotropic solids, J. Acoust. Soc. Am. 85 (1989) 2290-2300.

[43] P. Waterman, Orientation dependence of elastic waves in single crystals, Phys Rev. 113 (1959) 1240-1253, http://dx.doi.org/10.1103/PhysRev.113.1240.

[44] B. Kadomtsev, V. Petviashvili, On the stability of solitary waves in weakly dispersing media, Sov. Phys. Dokl. 15 (6) (1970) 539.

[45] E. Zabolotskaya, R. Khokhlov, Sov. Phys. Acoust. 15 (1969) 35.

[46] M. Ablowitz, D. Baldwin, Nonlinear shallow ocean-wave soliton interactions on flat beaches, Phys. Rev. E 86 (3) (2012) 036305.

[47] T. Kawasaki, S. Tamura, H. Maris, Multi-dimensional effects on the propagation of strain solitons in solids, J. Phys.: Conf. Ser. 92 (2007) 012091.

[48] T. Driscoll, A composite Runge-Kutta method for the spectral solution of semilinear PDE, J. Comp. Phys. 182 (2002) 357

[49] H. Maris, 6-Interaction of sound waves with thermal phonons in dielectric crystals, in: W. Mason, R. Thurston (Eds.), Principles and Methods, Physica Acoustics, vol. 8, Academic Press, 1971, p. 279. http://dx.doi.org/10.1016/ B978-0-12-395668-2.50011-9, <http://www.sciencedirect.com/science/ article/pii/B9780123956682500119>.

[50] B. Daly, N. Holme, T. Buma, C. Branciard, T. Norris, D. Tennant, J. Taylor, J. Bower, S. Pau, Imaging nanostructures with coherent phonon pulses, Appl Phys. Lett. 84 (25) (2004) 5180. <http://link.aip.org/link/?APL/84/5180/1>.

[51] A.G. Every, K.Y. Kim, A.A. Maznev, The elastodynamic response of a semiinfinite anisotropic solid to sudden surface loading, J. Acoust. Soc. Am. 102 (1997) 1346.

[52] O. Muskens, J. Dijkhuis, Interactions of ultrashort strain solitons and terahertz electronic two-level systems in photoexcited ruby, Phys. Rev. B 71 (10) (2005) 104304. <http://link.aps.org/abstract/PRB/v71/e104304>.

[53] A. Bartels, T. Dekorsy, H. Kurz, K. Köhler, Coherent zone-folded longitudinal acoustic phonons in semiconductor superlattices: excitation and detection, Phys. Rev. Lett. 82 (5) (1999) 1044, http://dx.doi.org/10.1103/PhysRevLett. 82.1044.

[54] B. Auld, Acoustic Fields and Waves in Solids, second ed., vol. 1, Robert E. Krieger Publishing Company, 1990.

[55] D. Dlott, New developments in the physical chemistry of shock compression, Annu. Rev. Phys. Chem. 62 (2011)

[56] D. Korteweg, G. de Vries, On the change of form of long waves advancing in a rectangular canal, and on a new type of long stationary waves, Phil. Mag. 39 (1895) 422.

[57] V. Karpman, Non-linear Waves in Dispersive Media, first ed., Pergamon Press, 1975.

[58] R. Bullough, P. Caudrey (Eds.), Solitons, Springer, 1980.

[59] J. Russell, Report on Waves, Report of the Fourteenth Meeting of the British Association for the Advancement of Science, 1844, p. 311.

[60] A. Osborne, T. Burch, Internal solitons in the Andaman Sea, Science 208 (1980) 451

[61] G. Ludwig, J. Ferreira, Y. Nakamura, Observation of ion-acoustic rarefaction solitons in a multicomponent plasma with negative ions, Phys. Rev. Lett. 52 (4) (1984) 275, http://dx.doi.org/10.1103/PhysRevLett.52.275.

[62] S. McCall, E. Hahn, Self-induced transparency, Phys. Rev. 183 (2) (1969) 457.

[63] L. Mollenauer, R.H. Stolen, J. Gordon, Experimental observation of picosecond pulse narrowing and solitons in optical fibers, Phys. Rev. Lett. 45 (13) (1980) 1095, http://dx.doi.org/10.1103/PhysRevLett.45.1095.

[64] D. Skyrabin, F. Luan, J. Knight, P. Russell, Soliton self-frequency shift cancellation in photonic crystal fibers, Science 301 (2003) 1705.

[65] J. Denschlag, J. Simsarian, D. Feder, C. Clark, L. Collins, J. Cubizolles, L. Deng, E. Hagley, K. Helmerson, W. Reinhardt, S. Rolston, B. Schneider, W. Phillips, Generating solitons by phase engineering of a Bose-Einstein condensate, Science 287 (1999) 97.

[66] K. Strecker, G. Partridge, A. Truscott, R. Hulet, Formation and propagation of matter-wave soliton trains, Nature 417 (2002) 150.

[67] R. Miurat, The korteweg-de Vries equation: a survey of results, SIAM Rev. 18 (3) (1976) 412.

[68] E.D. Palik (Ed.), Handbook of Optical Constants of Solids, Academic Press, Burlington, 2002. http://dx.doi.org/10.1016/B978-012544415-6.50145-X, <http://www.sciencedirect.com/science/article/pii/B978012544415650145X>.

[69] A. Huynh, N. Lanzillotti-Kimura, B. Jusserand, B. Perrin, A. Fainstein, M Pascual-Winter, E. Péronne, A. Lemaitre, Subterahertz phonon dynamics in acoustic nanocavities, Phys. Rev. Lett. 97 (11) (2006) 115502. <http:// link.aps.org/abstract/PRL/v97/e115502>.

[70] A. Scherbakov, P. van Capel, A. Akimov, J. Dijkhuis, D. Yakovlev, T. Berstermann, M. Bayer, Chirping of an optical transition by an ultrafast acoustic soliton train in a semiconductor quantum well, Phys. Rev. Lett. 99 (5) (2007) 057402 <http://link.aps.org/abstract/PRL/v99/e057402>.

[71] M. Bargheer, N. Zhavoronkov, Y. Gritsai, J. Woo, D.S. Kim, M. Woerner, T Elsaesser, Coherent atomic motions in a nanostructure studied by femtosecond X-ray diffraction, Science 306 (5702) (2003) 1771.

[72] A. Bojahr, M. Herzog, D. Schick, I. Vrejoiu, M. Bargheer, Calibrated real-time detection of nonlinearly propagating strain waves, Phys. Rev. B 86 (14) (2012) 144306.

[73] R. Tobey, M. Siemens, O. Cohen, M. Murnane, H. Kapteyn, K. Nelson, Ultrafast extreme ultraviolet holography: dynamic monitoring of surface deformation, Opt. Lett. 32 (3) (2007) 286

[74] S. Ayrinhac, A. Devos, A.L. Louarn, P.-A. Mante, P. Emery, Ultrafast acoustics in the middle uv range: coherent phonons at higher frequencies and in smaller objects, Opt. Lett. 35 (20) (2010) 3510, http://dx.doi.org/10.1364/ OL.35.003510. <http://ol.osa.org/abstract.cfm?URI=ol-35-20-3510>. 
[75] E. Reed, M. Armstrong, K.-Y. Kim, J. Glownia, Atomic-scale time and space resolution of terahertz frequency acoustic waves, Phys. Rev. Lett. 101 (1) (2008) 014302

[76] M. Armstrong, E. Reed, K.-Y. Kim, J. Glownia, W. Howard, E. Piner, J. Roberts, Observation of terahertz radiation coherently generated by acoustic waves, Nat. Phys. 5 (4) (2009) 285.

[77] D. Moss, A. Akimov, R. Campion, M. Henini, C. Foxon, L. Eaves, A. Kent, B. Glavin, Picosecond strain pulses probed by the photocurrent in semiconductor devices with quantum wells, Phys. Rev. B 83 (24) (2011) 245303.

[78] V. Temnov, Ultrafast acousto-magneto-plasmonics, Nat. Photon. 6 (11) (2012) 728.

[79] V. Temnov, C. Klieber, K. Nelson, T. Thomay, V. Knittel, A. Leitenstorfer, D. Makarov, M. Albrecht, R. Bratschitsch, Femtosecond nonlinear ultrasonics in gold probed with ultrashort surface plasmons, Nat. Commun. 4 (1468).

[80] A. Chabchoub, M. Fink, Time-reversal generation of rogue waves, Phys. Rev. Lett. 112 (2014) 124101, http://dx.doi.org/10.1103/PhysRevLett.112.124101.
[81] D. Hurley, O. Wright, O. Matsuda, V. Gusev, O. Kolosov, Laser picosecond acoustics in isotropic and anisotropic materials, Ultrasonics 38 (1-8) (2000) 470

[82] T. Dehoux, N. Chigarev, C. Rossignol, B. Audoin, Effect of lateral electronic diffusion on acoustic diffraction in picosecond ultrasonics, Phys. Rev. B 77 (21) (2008) 214307, http://dx.doi.org/10.1103/PhysRevB.77.214307. <http:// link.aps.org/abstract/PRB/v77/e214307>.

[83] J. Jäger, A.V. Scherbakov, T. Linnik, D. Yakovlev, M. Wang, P. Wadley, V. Holy, S Cavill, A. Akimov, A. Rushforth, M. Bayer, Picosecond inverse magnetostriction in galfenol thin films, Appl. Phys. Lett. 103 (3) (2013). http://dx.doi.org/ 10.1063/1.4816014, <http://scitation.aip.org/content/aip/journal/apl/103/3/ $10.1063 / 1.4816014>$.

[84] T. Pezeril, N. Chigarev, P. Ruello, S. Gougeon, D. Mounier, J.-M. Breteau, P. Picart, V. Gusev, Laser acoustics with picosecond collimated shear strain beams in single crystals and polycrystalline materials, Phys. Rev. B 73 (13) (2006) 132301. <http://link.aps.org/abstract/PRB/v73/e132301>. 